NBER WORKING PAPER SERIES

\title{
LEAVING NO ETHICAL VALUE BEHIND: TRIAGE PROTOCOL DESIGN FOR PANDEMIC RATIONING
}

\author{
Parag A. Pathak \\ Tayfun Sönmez \\ M. Utku Unver \\ M. Bumin Yenmez \\ Working Paper 26951 \\ http://www.nber.org/papers/w26951
NATIONAL BUREAU OF ECONOMIC RESEARCH
1050 Massachusetts Avenue
Cambridge, MA 02138
April 2020

We are grateful for input from several medical professionals. Nikhil Agarwal, Alex Rees-Jones, Robert Truog, Iván Werning, and Doug White provided helpful comments. Feedback from Eric Budish, David Delacrétaz, and Govind Persad was particularly valuable. The views expressed herein are those of the authors and do not necessarily reflect the views of the National Bureau of Economic Research.

NBER working papers are circulated for discussion and comment purposes. They have not been peerreviewed or been subject to the review by the NBER Board of Directors that accompanies official NBER publications.

(C) 2020 by Parag A. Pathak, Tayfun Sönmez, M. Utku Unver, and M. Bumin Yenmez. All rights reserved. Short sections of text, not to exceed two paragraphs, may be quoted without explicit permission provided that full credit, including $(\mathcal{O}$ notice, is given to the source. 
Leaving No Ethical Value Behind: Triage Protocol Design for Pandemic Rationing Parag A. Pathak, Tayfun Sönmez, M. Utku Unver, and M. Bumin Yenmez

NBER Working Paper No. 26951

April 2020

JEL No. D45,D47,I3

\begin{abstract}
Rationing of medical resources is a critical issue in the COVID-19 pandemic. Most existing triage protocols are based on a priority point system, in which a formula specifies the order in which the supply of a resource, such as a ventilator, is to be rationed for patients. A priority point system generates an identical priority ranking specifying claims on all units. Triage protocols in some states (e.g. Michigan) prioritize frontline health workers giving heavier weight to the ethical principle of instrumental value. Others (e.g. New York) do not, reasoning that if frontline workers obtain high enough priority, there is a risk that they obtain all units and none remain for the general community. This debate is pressing given substantial COVID-19 health risks for frontline workers. In this paper, we analyze the consequences of rationing medical resources through a reserve system. In a reserve system, resources are placed into multiple categories. Priorities guiding allocation of units can reflect different ethical values between these categories. A reserve system provides additional flexibility over a priority point system because it does not dictate a single priority order for the allocation of all units. It offers a middle-ground approach that balances competing objectives, such as in the medical worker debate. This flexibility requires attention to implementation, especially the processing order of reserve categories. We describe our model of a reserve system, characterize its potential outcomes, and examine distributional implications of particular reserve systems. We also discuss several practical considerations with triage protocol design.
\end{abstract}

\author{
Parag A. Pathak \\ Department of Economics, E52-426 \\ MIT \\ 50 Memorial Drive \\ Cambridge, MA 02142 \\ and NBER \\ ppathak@mit.edu
}

Tayfun Sönmez

Boston College

Department of Economics

140 Comm. Ave.

Chestnut Hill, MA, 02467

tayfun.sonmez@bc.edu

\author{
M. Utku Unver \\ Boston College \\ Department of Economics \\ 140 Comm. Ave. \\ Chestnut Hill, MA, 02467 \\ unver@bc.edu
}

M. Bumin Yenmez

Boston College

Department of Economics

140 Commonwealth Ave.

Chestnut Hill, MA 02467

bumin.yenmez@bc.edu 
"If you fail to plan, you are planning to fail."

- Benjamin Franklin

\section{Introduction}

The COVID-19 pandemic has inspired renewed attention to how medical resources are rationed. As of the writing of this paper, intensive care unit (ICU) beds and ventilators are among the two most important scarce resources. In the United States, as of March 2020, there were between $68,000-85,000$ ICU beds for the adult population. The total number of full-featured ventilators ranged between 72,000-82,000 including those in the Strategic National Stockpile, and there were roughly 100,000 additional partial-feature ventilators (Emanuel et al., 2020). Operating ventilators also requires respiratory therapists and other staff, and a large fraction of existing ventilator capacity is already deployed during non-emergency times 11 The U.S. Centers for Disease Control estimates that upwards of 21 million Americans will require hospitalization for COVID-19, and evidence from Italy suggests that 10-25\% of those hospitalized will require ventilators (Truog, Mitchell and Daley, 2020). While these forecasts evolve daily, Emanuel et al. (2020) argue it is unclear how quickly supply can meet demand even with recent efforts to increase production of these critical resources.

How to implement a fair rationing system during a pandemic presents a complicated question rife with ethical concerns. Medical ethicists have established several principles for rationing scarce medical resources in the case of influenza. Many states have proposed frameworks or explicit rules during severe pandemics too $2^{2}$ These principles echo formal normative criteria in resource allocation problems in economics. For example, one principle is to treat people equally regardless of their socioeconomic status, age, or race. A second principle is to maximize expected health outcomes measured through age, preexisting health conditions, or some notion of quality-adjusted life years. A third, sometimes conflicting, principle is to help the worst off or the sickest. Yet another principle considers a patient's instrumental value, which is a forwardlooking justification for protecting frontline health workers. Whatever principle is adopted, there is widespread consensus that any rationing protocol should be transparent, applied consistently, and not be left to the discretion of frontline health workers, who may be under severe pressure (Truog, Mitchell and Daley, 2020).

Contested debates on these ethical principles have motivated several in the medical community to propose a priority point system. In a priority point system, an explicit formula specifies the order in which resources should be rationed. Although our analysis applies to a variety of medical resources, we use ventilators to simplify terminology.

The 2015 New York State Task Force on Ventilator Allocation is an example of a priority point system. It has influenced the protocols of several states. The system recommends that after

\footnotetext{
${ }^{1}$ The New York State Ventilator Guidelines in 2015 state that during non-emergency normal conditions, 85\% of ventilators are used, leaving only $15 \%$ available for new cases (Zucker et al. 2015, p. 27).

${ }^{2}$ Guidelines include Maryland (Daugherty-Biddison et al. 2017), Michigan (Michigan, 2012) Minnesota (Vawter et al. 2010), New York (Zucker et al., 2015), North Carolina (Tong and Devlin, 2007), Ontario (Christian et al., 2006), South Carolina (Ball and Schneider, 2009), the Swiss Influenza Pandemic Plan (Iten and Strupler, 2018), and Utah (DoH, 2018).
} 
certain patients are excluded, there should be a priority order for patients based on estimated mortality risk and such patient's priority status should be re-evaluated every 48 hours based on changes in health status (Zucker et al. 2015). Mortality risk is measured by the Sequential Organ Failure Assessment (SOFA) score $3^{3}$ At any priority level, there is the potential that one priority group could completely exhaust the remaining available resources. In cases of excess demand upon remaining resources by a given priority group, New York and other proposals recommended random allocation - a lottery - among equal-priority patients (Zucker et al., 2015; Emanuel et al. 2020).

Several criticize priority point systems solely based on SOFA for ignoring multiple ethical values. Task forces commissioned to develop guidelines recognize the need to integrate a variety ethical values and advocate for a multi-principle approach, see, e.g., White et al. (2009) and Daugherty-Biddison et al. (2017). For example, a Johns Hopkins study examining public perceptions of different ethical principles summarized (Biddison et al., 2013):

Both groups suggested alternative strategies, such as organ transplantation allocation criteria as a model or adopting a tiered approach by applying different principles at different stages in process.

To integrate multiple ethical values, White et al. (2009) formulates a multi-principle priority point system. Using a similar aggregation methodology as was used to construct the SOFA score, in this mechanism several ethical values are put on a numeric scale and summed up across ethical values to arrive at a single number. A patient with the lowest score receives the highest priority. Whereas the SOFA score is widely accepted as a proxy for mortality risk, aggregating scores across different ethical values may result in non-commensurable comparisons.

While a priority point system - whether single or multi-principle- does provide a well-defined protocol, we argue that it does not offer the flexibility to accommodate competing objectives and values. In this paper, we consider an alternative way to accommodate different ethical principles through a reserve system. In a reserve system, patients are identified as members of particular groups - e.g., young or old, frontline health worker or not, very sick or sick. Group membership can overlap: a young patient could also be a frontline health worker. In a reserve system, reserve sizes (i.e., the quantity of resources reserved) are set for each group, and within a reserve group, priority can be based on an explicit score or random assignment. Though the word reserve might suggest that some resources may be unused, in our main application of allocation of vital medical resources, we restrict attention to soft reserves where no units are left unassigned 4 A reserve system provides additional flexibility over a priority point system because priority points need not apply to the allocation of all resources. For example, a fraction is reserved for frontline health workers, while the rest is unreserved for all community members. Frontline health workers are always first in line in their reserve category, and the remaining

\footnotetext{
${ }^{3}$ The European Society of Intensive Care Medicine devised the SOFA score at a consensus meeting in October 1994 in Paris, France (Vincent et al., 1996). Each of six organ systems - lungs, liver, brain, kidneys, blood clotting, and blood pressure - is independently assigned a score of 1 to 4 . The SOFA score sums these six scores, with sicker patients generally being assigned higher scores. While not initially designed as a prognostic score, subsequent research supports its use for that end (Jones, Trzeciak and Kline, 2009).

${ }^{4}$ See Section 3.4 for the definition of soft and hard reserves.
} 
priorities can be the same. This system balances their interests against other ethical goals.

Reserve systems are widespread in resource allocation settings outside of medicine when there are conflicting objectives 5 The key idea of a reserve system is to divide the total supply into several categories, and consider allocation for these smaller number of units separately. Specific objectives can be realized within these categories, using explicit priorities or randomization. The advantage of a reserve system is in its flexibility. A priority point system obscures trade-offs between different principles because it aggregates several different considerations into a single priority score. It is even possible that one principle might dominate other principles unexpectedly depending on how scores are scaled.

It is important to note that we are agnostic on what the reserve types or sizes should be. Our primary aim is to inform the debate on how a reserve system can be used to balance competing objectives, and provide a route forward in several high-stakes debates on rationing. We do, however, discuss some reserve categories below only to indicate possibilities, and leave the final decisions to medical ethicists, task forces, and other stakeholders.

We first illustrate the power of a reserve system by explaining how it can assist with the debate on whether frontline health workers should obtain priority for vital medical resources. Under Michigan's guidelines, essential personnel are prioritized for these resources (Michigan, 2012). Ethicists have also emphasize its importance in the current pandemic (see, e.g., Emanuel et al. (2020) and White and Lo (2020). On the other hand, the Minnesota Pandemic Working group argued against prioritizing frontline health workers, suggesting they should be treated the same way as all other patients (Vawter et al., 2010). One reason they offer is

... it is possible that they [key workers] would use most, if not all, of the short supply of ventilators; other groups systematically would be deprived access.

The New York State Task Force also struggled with this topic, recognizing the need to provide "insurance" for frontline health workers. However, they ultimately decided that access should only depend on a patient's clinical factors and not their occupation. There are presently deep disagreements on these issues as several states, medical associations, and hospitals develop their own guidelines (Fink, 2020). A reserve system provides a resolution to this conundrum, because only a subset of resources are reserved for frontline health workers and the rest are unreserved and open for any group that is eligible for the resources. Prioritization within these two categories can be based on a lottery or explicit criteria related to mortality risk. A reserve system would accommodate both concerns.

A second debate about priority systems involves whether a certain group can be excluded from treatment as opposed to merely being placed in the lowest priority group. In the Alabama plan announced in March 2020, individuals with severe or profound mental disabilities were considered "unlikely candidates for ventilator support." [6 Washington state guidelines recommended that hospital patients with "loss of reserves in energy, physical ability, cognition and general health" be switched to outpatient or palliative care (Fink, 2020). If certain groups are

\footnotetext{
${ }^{5}$ Examples include school assignment, affirmative action, and immigration visa allocation.

${ }^{6}$ After widespread backlash, this plan was withdrawn on April 9, 2020. Carter (2020) warns that plans that discriminate against the disabled may violate the Americans with Disabilities Act.
} 
prohibited from receiving vital resources, it violates a fundamental principle of non-exclusion. That is, it violates the idea that every patient, no matter his or her circumstances, should have some hope of obtaining a life-saving resource. In a reserve system, if a portion of vital resources are set aside for all, even if that allotment is small, and random allocation is used for that segment, every patient could possibly obtain it. In contrast, in a priority system coupled with excess demand for available resources by the higher-priority groups - even without any explicit exclusion of certain types of individuals - there will be some patients who would never be treated during a shortage.

Reserve systems have an important medical precedent in deceased donor kidney allocation, where there is also scarcity. Until 2014, the U.S. Organ Procurement and Transplantation Network (OPTN) used a priority system to allocate deceased donor kidneys (OPTN, 2014). After establishing medical compatibility, patients were ordered by priority type, and then priority points, with ties broken by waiting time 7 In 2014, the OPTN system changed to include a reserve (Israni et al., 2014). In the reserve, $20 \%$ of the highest quality kidneys are reserved for adults with the highest $20 \%$ expected post-transplant survival score (EPTS). A priority points system is used for the remaining kidneys. Parallel to our motive for introducing reserve systems (i.e. better integration of various ethical principles), OPTN added this reserve to better reflect the ethical principle of utilitarianism, which was not as prominently captured in the prior system 8

While the advantage of a reserve system is its flexibility, it requires careful implementation. In some cases, implementation is straightforward. For kidneys, specific kidneys are reserved based on their quality through a formula, and there is a natural order of awarding these kidneys based on their arrival time. That is, whenever a high-quality kidney that qualifies for the $20 \%$ reserve arrives, priority is given to patients in the preferential treatment group for these types of kidneys. However, for rationing homogenous objects like ventilators or ICU beds, the design of reserve systems is more challenging. In particular, in a system that reserves a fraction of units for various groups, the processing order of reserve categories is important. In fact, there are now several instances of reserve systems with systematic implementation errors because of these complexities. $9^{9}$

Since a reserve system is not uniquely defined in our application, we start our theoretical analysis by formulating three basic principles any reserve system should satisfy. Theorem 1 characterizes the entire class of reserve systems satisfying these properties. Some mechanisms in this class have intuitive interpretations, while others do not. Given our market design focus, we restrict attention on a subset of these mechanisms which have clear interpretations and have the greatest practical relevance. These mechanisms process reserve categories sequentially and are widely observed in real-life applications. A major insight of our analysis is that when implementing a reserve system, processing a reserve category earlier is detrimental to its beneficiaries (Theorem 2). Theorems 3 and 4 establish upper and lower bounds for certain groups of

\footnotetext{
[Agarwal et al. (2019) describe that in the NYC-area system, priority was given first to patients with a perfect tissue-type match, then to patients from the local procurement organization, and finally to patients in close geographic proximity.

${ }^{8}$ The New Kidney Allocation System (KAS) Frequently Asked Questions, Organ Procurement and Transplantation Network. Available at https://optn.transplant.hrsa.gov/media/1235/kas_faqs.pdf

${ }^{9}$ See Section 2.3
} 
individuals for the case of hard reserves.

Section 2 discusses several practical aspects of triage protocol design and its connection to the COVID-19 pandemic. Section 3 presents our formal model of reserve systems, states our main characterization theorem, and analyzes the welfare implications of reserve processing. Section 4 discusses some other considerations for triage rationing as well as some future research directions. The paper concludes in Section 5. All proofs are relegated to the appendix.

\section{Design Considerations for Triage Protocol}

\subsection{The Case Against the Priority Point System}

In a priority point system, all units are allocated following a single priority order, often determined with points. Mortality risk, often measured by SOFA scores, is a common input to the point score. In some states, the priority system is only based on SOFA scores. White et al. (2009) criticize the use of these single-principle priority point systems for a narrow utilitarian perspective that completely ignores several other ethical values. This criticism is also echoed by several community studies including Biddison et al. (2013) and Daugherty-Biddison et al. (2017). White et al. (2009) advocates a multi-principle approach and formulates a multi-principle priority point system to implement this approach. This system, shown in Table 1, has become the leading multi-principle priority point system 10

Table 1. Illustration of Multi-Principle Strategy for Ventilator Allocation

\begin{tabular}{|c|c|c|c|c|c|}
\hline \multirow[t]{2}{*}{ Principle } & \multirow[t]{2}{*}{ Specification } & \multicolumn{4}{|c|}{ Point System } \\
\hline & & 1 & 2 & 3 & 4 \\
\hline $\begin{array}{l}\text { Save the } \\
\text { most lives }\end{array}$ & $\begin{array}{l}\text { Prognosis for short } \\
\text { term survival (SOFA } \\
\text { score) }\end{array}$ & SOFA $<6$ & SOFA 6-9 & SOFA $10-12$ & SOFA $>12$ \\
\hline $\begin{array}{c}\text { Save the } \\
\text { most years } \\
\text { of life }\end{array}$ & $\begin{array}{c}\text { Prognosis for long } \\
\text { term survival (Medical } \\
\text { assessment of } \\
\text { comorbidities) }\end{array}$ & $\begin{array}{c}\text { No } \\
\text { comorbidities }\end{array}$ & $\begin{array}{c}\text { Minor } \\
\text { comorbidities }\end{array}$ & $\begin{array}{c}\text { Major } \\
\text { comorbidities }\end{array}$ & $\begin{array}{l}\text { Severe } \\
\text { comorbidities; } \\
\text { likely death } \\
\text { within } 1 \text { year }\end{array}$ \\
\hline $\begin{array}{l}\text { Life-cycle } \\
\text { principle }\end{array}$ & $\begin{array}{l}\text { Prioritize those who } \\
\text { have had the least } \\
\text { chance to life through } \\
\text { life's stages }\end{array}$ & Age $12-40$ & Age $41-60$ & Age $61-74$ & Age $>74$ \\
\hline
\end{tabular}

Notes: SOFA stands for sequential organ failure assessment. Multi-principle point system developed by White et al. (2009)

Under the White et al. (2009) point system, the ethical values of saving the most lives, saving the most years of life, and the life-cycle principle are integrated through an additive formula. To

\footnotetext{
${ }^{10}$ As of April 24, 2020, California, Colorado, Massachusetts, New Jersey, Oklahoma, and Pennsylvania have adopted variants of this system. de Pu Kamp, Devine and Griffin (2020) reports that several hundred hospitals around the country have adopted this system.
} 
illustrate how it works, consider a hypothetical patient with a SOFA score of seven. She obtains two points based on the ethical value of saving the most lives. If the patient has no chronic diseases or comorbidities and is between 61-74 years old, she obtains four more points based on the other two ethical values yielding a total of six. A patient with a lower total point score has a higher priority for the resource than a patient with a higher total point score ${ }^{11}$ Between the SOFA-based priority point system and White et al. (2009) multi-principle point system, more than half of US states use a priority point system (Whyte, 2020).

The strength of the priority point mechanism is simplicity. Each ethical value is represented with a monotonic integer valued function. Values are then integrated with an additive formula producing an aggregate point score for each patient. The claims of patients over medical resources are determined based on their point scores, with a lower score typically indicating a higher claim. While practical, priority point mechanisms are limiting for a number of reasons.

First, priority points norm or scale different and potentially-unrelated ethical principles into one dimension. These challenges are like the usual ones associated with aggregating social alternatives into a single ordering based on multiple inputs - a situation which involves "comparing apples to oranges." The debate on how rationing guidelines should compare claims of children versus adults illustrates the issue. Massachusetts guidelines state that indicators that feed into scores for adults are not reliable for children (Bateman et al., 2020). They explain that "scoring systems that are meaningful for adult critical care patients do not apply to pediatric patients or newborns." As a result, the Massachusetts guidelines use a different scoring system for children. However, their point system then uses a single priority point system to evaluate all patients together, which ends up comparing the point scores of children with those of adults.

Second, there are some ethical principles where the claims of patients cannot be represented with a monotonic function. One example pertains to group-based policies, such as those related to regions or gender. For example, the European Union has proposed balanced participation of women and men in political and public decision making by requiring that at least $40 \%$ of public offices are held by women and at least $40 \%$ are held by men (Dittmar, 2018; Rankin, 2020). For medical rationing, it is possible that a future pandemic is so devastating that it threatens a significant portion of the human race. In such a hypothetical crisis, a principle based on survival of the species may suggest a similar constraint. A guideline may recommend to allocate at least $40 \%$ of vital resources to female patients and at least $40 \%$ to male patients. Clearly, considerations based on group composition cannot be represented with a function that relies only on individual attributes. Similarly, a priority point system cannot accommodate a guideline that wishes to allocate resources to disabled citizens in proportion to their representation in society.

The visible debate on prioritizing essential personnel also illustrates problems with priority point systems. Claims for essential personnel are based on the backward-looking principle of reciprocity and the forward-looking principle of instrumental value, which have been strongly endorsed by medical ethicists (Emanuel et al. 2020). Despite recognizing these claims, state task forces in New York and Minnesota ultimately did not embrace them. They cited concerns about the extreme scenarios where no units remain for general community members (Zucker et al., 2015.

\footnotetext{
${ }^{11}$ Most protocols specifies a tie-breaker between patients with identical total points, although the South Carolina protocol fails to provide one.
} 
Vawter et al., 2010). In contrast, priority is given to essential workers in states like Michigan. In between, some guidelines recognize priority for this group, but are vague on implementation. For example, Massachusetts guidelines provide precise details about its adaption of White et al. (2009). Yet, their guidelines append a paragraph calling for "heightened priority" for frontline workers without describing how it is to be implemented. This lack of transparency contrasts with the level of precision regarding other ethical principles, and may reflect their inability to arrive at consensus given the underlying priority point system. These examples show the restriction to priority point systems has often resulted in adaptation of two polar opposite policies on essential personnel priority, or an imprecise description of it.

\subsection{Reserve Systems as a Remedy and Its Main Parameters}

All of the challenges presented in Section 2.1 stem from one simple but limiting feature of a priority point system: it results in a priority ranking that is identical for each unit. A reserve system eliminates this feature of the mechanism because it allows for heterogeneity of patient claims over different units.

A reserve system has three main parameters. They are:

1. a division of all units into multiple segments referred to as reserve categories 12

2. number of units in each of these categories, and

3. specification of a priority order of the patients in each of these categories.

For some (or all) of the reserve categories, there can also be exclusion criteria, based on the nature of the medical resource that is being rationed along with the patient's clinical assessment. The priority order of patients for each category also incorporates this information. Reserve categories can differ either based on the groups to receive higher priority, or the combination of ethical principles to be invoked. The main idea is to use the associated priority order - which embeds ethical principles - when allocating units in each reserve category. Importantly, the priority order need not be the same between reserve categories.

In some reserve systems, three conditions hold: 1) units are heterogeneous and specific units are reserved for specific reserve categories, 2) units are perishable and have to be allocated as soon as they arrive, and 3) units arrive sequentially. Under these conditions, the allocation process is uniquely determined: allocate each unit as soon as it arrives to the highest priority agent in its reserve category. These conditions apply, for example, for the reserve system used for the allocation of deceased-donor kidneys in the U.S.

In many applications, however, one or more of these conditions fail. When this happens, there are several mechanisms to implement a reserve system. Our application, the allocation of medical resources during a pandemic, is an example where three conditions are not satisfied and there are multiple ways to implement a reserve system. While scarce medical resources may become available sequentially, they are often homogenous and non-perishable. A reserve system can be implemented in those applications through a wide class of mechanisms, formally characterized in Theorem 1 in Section 3. The outcome of these mechanisms can differ considerably

\footnotetext{
${ }^{12}$ This division is for accounting purposes only, and it does not attach a specific unit to a category.
} 
depending on the choice of the mechanism, and not all of these mechanisms have an intuitive interpretation. This multiplicity resulted in the emergence of a subclass of these mechanisms in real-life applications of these problem, where categories are processed sequentially for a given order of categories. In the context of medical resource rationing, our focus is this intuitive subclass of reserve systems we call sequential reserve matching rules. As a result, for our main application of interest, there is one additional parameter of a reserve system: the processing sequence of categories. This parameter plays an important role in the distribution of the units, and so we elaborate on its relevance next.

\subsection{Reserve Category Processing Sequence}

Sequential reserve matching rules were first formally introduced by Kominers and Sönmez (2016) in a more general environment with heterogenous units and multiple terms of allocation. Although not life-and-death situations, reserve systems are widespread in real-life applications including the implementation of affirmative action policies in school choice in Boston (Dur et al. 2018), Chicago (Dur, Pathak and Sönmez, 2019), the implementation of reservation policies in India (Sönmez and Yenmez, 2019a,b), and the allocation of immigration visas in the U.S. (Pathak, Rees-Jones and Sönmez, 2020a). As shown in these studies, the processing order of reserve categories is a key parameter with significant distributional implications ${ }^{13}$

To explain intuitively why processing order is important, imagine a simple scenario in which there are 60 ventilators. A medical ethics committee decides that there are two important principles: equal treatment of equals and prioritizing essential medical personnel. Based on their view, they define a reserve category for essential medical personnel, which reserves $50 \%$ of ventilators for them. Within this reservation, there is random allocation via lottery. The remaining $50 \%$ of ventilators are unreserved and open to all patients, including essential personnel. These are also allocated via lottery. Suppose that there are 60 essential personnel who need a ventilator and 60 other patients who do as well. If the essential personnel reserve category is processed first, then all 30 ventilators in this category are allocated to them. If a new lottery is drawn for the remaining 30 unreserved ventilators, since there are 60 other patients and 30 remaining essential personnel, the lottery results in 20 unreserved ventilators $(2 / 3 \times 30=20)$ allocated to other patients and 10 unreserved ventilators $(1 / 3 \times 30=10)$ to essential personnel. The total number of ventilators awarded to essential personnel is 40 and the total number awarded to other patients is 20 . On the other hand, if the reserve category is processed after the unreserved units, then the 30 unreserved units will be split evenly between the essential personnel and other

\footnotetext{
${ }^{13}$ The U.S. Customs and Immigration has a reserve system for the H1-B immigration visas, and since 2005, it has deployed four different allocation mechanisms, sometimes unintentionally (Pathak, Rees-Jones and Sönmez, 2020a). Boston Public Schools had a reserve system in its school assignment plan in which half of each school's seats were reserved for students from the neighborhood. In 2013, after realizing that how reserves are processed dramatically influences their impact, the city abandoned the reserve after significant public debate (Pathak and Sönmez, 2013b, Dur et al., 2018). India's public universities and jobs use reserve systems for affirmative action. Since there are many traits eligible for reserves, faulty implementation of reserves has generated countless litigation and confusion (Sönmez and Yenmez, 2019ab). Finally, Pathak, Rees-Jones and Sönmez $(2020 b)$ show that a large number of participants in an incentivized experiment have incorrect beliefs about some properties of reserve systems.
} 
patients. The 30 remaining ventilators are all reserved and allocated to essential personnel. This results assigning 45 ventilators to essential personnel and 15 ventilators to other patients. Thus in this simple example, the choice of the processing sequence of categories is a matter of life or death for 5 essential medical personnel and 5 members of the general community.

As this simple example illustrates, our application to triage protocol design is another setting where reserve processing matters. Indeed, understanding the implications of reserve category processing order is especially critical in our application given the emphasis on transparency. Much of our theoretical analysis in Section 3 relates to this subtle aspect of sequential reserve matching rules. Perhaps the most important lesson from this analysis is that the later a reserve category is processed the better it is for its beneficiaries. This important feature, also apparent in the example above, has the following important implications for design. If a reserve category is intended as a "boost" for a group of participants, then the category should be processed after more inclusive categories open to all. This form of implementing reserve policies can be interpreted as an over-and-above policy. In contrast, if a reserve category is intended as a "protective measure" for a group of participants, then the category should be processed after more inclusive categories open to all. This second form of implementing reserve policies can be interpreted as a minimum guarantee policy 14

\subsection{Potential Reserve Categories}

The parameters of a reserve system can be modified for different medical resources. Emanuel et al. (2020) emphasizes that "prioritization guidelines should differ by intervention and should respond to changing scientific evidence." In particular, vaccines and tests may rely on different ethical principles than ventilators and ICU beds. Clearly, decisions about reserve sizes and reserve categories are up to the medical community and other stakeholders. However, we next describe some potentially important reserve categories that can be utilized to overcome several difficulties that emerge due to limitations of priority point systems.

Any reserve category has to be based on well-established ethical principles. One of these principles is reciprocity towards persons who accept risk for the common good of saving lives or who have saved lives in the past. Reciprocity is a backward-looking principle and is closely related to the forward-looking principle of instrumental value, which gives priority to those who can save others in the future (Emanuel et al., 2020, White and Lo, 2020).

If the intention of a reserve category is based on either or both of these ethical values, then the category could be implemented in the form of over-and-above policies to provide an additional boost. The first reserve category we consider below, motivated by the real-life difficulties of implementing essential personnel priorities under existing guidelines, is based on both ethical

\footnotetext{
${ }^{14}$ Starting with the landmark Supreme Court of India case Indra Sawhney and others v. Union of India (1992), over-and-above and minimum guarantee policies have been explicitly considered in countless court decisions on affirmative action in India. The judges in Indra Sawhney (1992) introduced the over-and-above implementation as a higher form of reserve policies to provide an additional boost to the historically discriminated against members of the reserve groups such as Scheduled Castes, Scheduled Tribes and Other Backward Classes, and they introduced the minimum guarantee implementation as a lower form of these policies as a protective measure for other disadvantaged groups such as persons with disabilities. Sönmez and Yenmez (2019a $b)$ conduct a detailed analysis of Indian affirmative action policies.
} 
principles.

\subsubsection{Essential Personnel Category}

The essential personnel category provides some form of priority to personnel such as frontline health workers. Essential personnel may have made potentially life-saving contributions to society in the past, and they are presently subject to severe risks. Therefore, ethicists justify

this reserve on the basis of both reciprocity and instrumental value. Furthermore, Emanuel et al. (2020) offers the following incentive-based rationale for prioritizing essential personnel:

... but giving them priority for ventilators recognizes their assumption of the high-risk work of saving others, and it may also discourage absenteeism.

Nevertheless, essential personnel are not prioritized in several state guidelines. One of the main justifications for denying essential personnel priority is articulated in 2015 New York State Ventilator Allocation Guidelines (Zucker et al., 2015):

Expanding the category of privilege to include all the workers listed above may mean that only health care workers obtain access to ventilators in certain communities. This approach may leave no ventilators for community members, including children; this alternative was unacceptable to the Task Force.

Limiting priority allocation of ventilators to essential personnel for only a subset of ventilators is a natural compromise, compared to the two extreme policies that either provide it for all units (e.g. Michigan) or for none of the units (e.g. New York State and Minnesota).

\subsubsection{Good Samaritan Reciprocity Category}

Another possible category is a Good Samaritan reciprocity category, which provides priority based on Good Samaritan acts. In such a category, a small fraction of resources are reserved for those who have saved lives through their past Good Samaritan acts. These could be participants for clinical trials on vaccine or treatment development (Emanuel et al., 2020), altruistic donors who have donated their kidneys to a stranger, or people who have donated large quantities of blood over the years. Good samaritan status can also be provided for compatible patient-donor pairs who voluntarily participate in kidney exchange even though they do not have to, and save another patient's life who was incompatible with his/her donor. This type of incentive could save a large number of lives. Sönmez, Ünver and Yenmez (2020) estimate a 180 additional kidney patients could receive living donor transplants for every 10 percent of compatible pairs who can be incentivized to participate in kidney exchange. A state task force can determine which acts "deserve" a Good Samaritan status.

In addition to the widely-accepted ethical principle of reciprocity, this category can also be motivated by the incentives it creates. If the aim is to maximize this incentive, it could be implemented as an over-and-above policy. Even though a triage rationing protocol will hopefully be rarely deployed, the mere existence of a modest reserve of this nature may mitigate more persistent and ongoing crises in other healthcare domains through the incentives it creates. 


\subsubsection{Protective Reserve Categories: Disabled and Disadvantaged}

Disabilities rights advocates have opposed rationing plans based on expected health outcomes using survival probabilities because such criteria are inherently discriminatory ${ }^{15}$ Persad $(2020)$ recounts that several prefer either random selection or minimal triage that completely ignores any differences in likelihood or magnitude of benefit, or the likely quantity of resources required for benefit. A reserve system allows for a resolution of this dispute. In particular, a disabled protective category can be established for disabled patients reserving some of the units for these groups. If the representatives of these groups reach a decision to implement random lottery within disabled patients for these units, this can be implemented under a reserve system without interfering with the priority order for units in other categories.

Another criticism of priority point systems which use mortality risk or comorbidities as part of the priority score is that these criteria do not take into account differences in expected health outcomes driven by discrimination in access to health care or other social inequalities. For instance, disparate access to testing for disadvantaged groups may increase COVID-19 prevalence in these communities (Blow, 2020). A reserve system can be used to accommodate this perspective. A portion of scarce resources could be set aside in the form of a disadvantaged protective category based on legally-permissible measures of disadvantage. If this reserve is intended solely for protective reasons as we envision, it could be implemented as a minimum guarantee.

\subsection{Priorities}

The last lever of a reserve system is the priority order in each reserve category. As we have already described, each of the potential categories we discussed, i.e. essential personnel category, Good Samaritan reciprocity category, disabled protective category, or disadvantaged protective category would prioritize the target group of patients relative to others. However, the extent to which policymakers prioritize patients from groups relative to others is flexible. We offer a few examples to show possibilities.

If instrumental value is the primary ethical consideration, the essential personnel reserve category could give absolute priority to frontline health workers and clinical criteria could be used as a tie-breaker in this category. Alternatively, clinical criteria could be the main criteria, and essential personnel status could be a tie-breaker. Both methods are valid in a reserve system, although with different degrees of preferential treatment. In fact, this example is similar to an idea explored in Pittsburgh's system (White et al., 2020). Although there is no reserve, the priority score uses the SOFA score and comorbidities. Life-cycle and instrumental considerations shape the tie-breakers:

In the event that there are 'ties' in priority scores/categories between patients and not enough critical care resources for all patients with the lowest scores, life-cycle considera-

\footnotetext{
${ }^{15}$ Ne'eman (2020) argues that provisions that exclude certain groups can undermine overall trust in the medical system "based on a well-founded fear of being sacrificed for the greater good." The Disability Rights Education and Defense Fund argues that the probability of survival should not be considered as long as it is positive (DREDF 2020). That is, they claim that the "use of a disability to deny or limit an individual's access to health care or to provide them a lower priority in accessing scarce resources or supplies constitutes a clear violation of disability nondiscrimination law."
} 
tions should be used as the first tiebreaker, with priority going to younger patients. We recommend the following categories: age 12-40, age 41-60, age 61-75, older than age 75. We also recommend that individuals who are vital to the acute care response be given priority, which could be operationalized in the form of a tiebreaker.

The Pittsburgh system illustrates that preferential treatment for essential personnel can be modest: it is only a tie-breaker and the primary ethical considerations are based on saving the most lives 16

\section{Formal Model and Analysis}

While our primary application is pandemic rationing of scarce medical resources, in this section we provide a more general model which has several other applications including affirmative action in school choice (Pathak and Sönmez, 2013b, Dur et al., 2018; Correa et al., 2019), college admissions (Aygün and Bo, 2016, Baswana et al., 2018), assignment of government positions (Sönmez and Yenmez, 2019a b), and diversity in immigration visa allocation (Pathak, Rees-Jones and Sönmez, 2020a). Despite this generality, the terminology is tailored to our main application.

There is a set of patients $I$ and $q$ identical medical units to allocate. There is a set of reserve categories $\mathcal{C}$. For each category $c \in \mathcal{C}, r_{c}$ units are reserved so that $\sum_{c \in \mathcal{C}} r_{c}=q$. It is important to emphasize that individual units are not associated with the categories in our model. The phrase " $r_{c}$ units are reserved" does not mean specific units are set aside for category $c$. Rather, it means that for the purposes of accounting, a total of unspecified $r_{c}$ units are attached to category $c$.

For each category $c \in \mathcal{C}$, there is a linear priority order $\pi_{c}$ over the set of patients $I$ and $\emptyset$. For our main application of pandemic rationing, the linear order $\pi_{c}$ priority orders the patients based on the ethical principle guiding allocation of units in category $c$. For each category $c \in \mathcal{C}$ and patient $i \in I$, we say that patient $i$ is eligible for category $c$ if

$$
i \pi_{c} \emptyset \text {. }
$$

A matching $\mu: I \rightarrow \mathcal{C} \cup\{\emptyset\}$ is a function that maps each patient either to a category or to $\emptyset$ such that, $\left|\mu^{-1}(c)\right| \leq r_{c}$ for every category $c \in \mathcal{C}$. For any patient $i \in I, \mu(i)=\emptyset$ means that the patient does not receive a unit and $\mu(i)=c \in \mathcal{C}$ means that the patient receives a unit reserved for category $c$. Let $\mathcal{M}$ denote the set of matchings.

For any matching $\mu \in \mathcal{M}$ and any subset of patients $I^{\prime} \subseteq I$, let $\mu\left(I^{\prime}\right)$ denote the set of patients in $I^{\prime}$ who are matched with a category under matching $\mu$. More formally,

$$
\mu\left(I^{\prime}\right)=\left\{i \in I^{\prime}: \mu(i) \in \mathcal{C}\right\} .
$$

\footnotetext{
${ }^{16}$ The Pittsburgh system also suggests the potential need for a reserve system, given that its designers hesitated recommending a single tie-breaker. Instead, the guideline suggests utilization of either the age or essential personnel status as a tie-breaker. Clearly, the designers saw saving the most lives as more justified than either the life-cycle principle or the instrumental value principle. On the other hand, the guidelines did not choose between these two ethical values in the event of tie-breaking. In contrast, a reserve system offers flexibility to use one of these tie-breakers in one of the categories and the other in another category.
} 
These are the patients in $I^{\prime}$ who receive units under matching $\mu$.

In real-life applications of our model, it is important to allocate units to qualified individuals without wasting any, and following the priorities attached to these units. We next formulate this idea through three axioms:

Definition 1 A matching $\mu \in \mathcal{M}$ is individually rational if, for any $i \in I$ and $c \in \mathcal{C}$,

$$
\mu(i)=c \quad \Longrightarrow \quad i \pi_{c} \emptyset \text {. }
$$

Our first axiom formulates the idea that individuals should only receive those units for which they are qualified. That is, units should be awarded only to eligible individuals. For ventilator rationing, any patient who is eligible for one category must also be eligible for any category. And if a patient is ineligible for all categories, then this patient can be dropped from the set of patients. Hence, individual rationality always holds for our main application.

Definition 2 A matching $\mu \in \mathcal{M}$ is non-wasteful if, for any $i \in I$ and $c \in \mathcal{C}$,

$$
i \pi_{c} \emptyset \quad \text { and } \quad \mu(i)=\emptyset \quad \Longrightarrow \quad\left|\mu^{-1}(c)\right|=r_{c} .
$$

Our second axiom formulates the idea that no unit should be wasted. That is, if a unit remains idle, then there should not be any individual who is both unmatched and also qualified for the unit. For rationing vital resources, each patient is eligible for all units, and therefore non-wastefulness in this context corresponds to either matching all the units or all the patients.

Definition 3 A matching $\mu \in \mathcal{M}$ respects priorities if, for any $i, i^{\prime} \in I$ and $c \in \mathcal{C}$,

$$
\mu(i)=c \text { and } \mu\left(i^{\prime}\right)=\emptyset \quad \Longrightarrow \quad i \pi_{c} i^{\prime} .
$$

Finally our last axiom formulates the idea that for each category, the units should be allocated based on the priority order of individuals in this category.

It is important to emphasize that in many real-life rationing guidelines each of these axioms is implicit. Our formulation differs from these real-life guidelines only in its flexibility to allow for heterogeneity in priority orders guiding allocation of individual units.

\subsection{Main Characterization Result}

In this section, we present a full characterization of all matchings that satisfy the three axioms formulated above. The characterization utilizes the celebrated deferred-acceptance algorithm of Gale and Shapley (1962).

Consider the following hypothetical many-to-one matching market. The two sides of the market are the set of patients $I$ and the set of categories $\mathcal{C}$. Each patient $i \in I$ can be matched with at most one category, whereas each category $c \in \mathcal{C}$ can be matched to as many as $r_{c}$ patients. Category $c$ is endowed with the linear order $\pi_{c}$ that is specified in the primitives of the original rationing problem. 
Observe that in our hypothetical market, all the primitives introduced so far naturally follows from the primitives of the original problem. The only primitive of the hypothetical market that is somewhat "artificial" is the next one:

Each patient $i \in I$ has a strict preference relation $\succ_{i}$ over the set $\mathcal{C} \cup\{\emptyset\}$, such that, for each patient $i \in I$,

$$
c \succ_{i} \emptyset \quad \Longleftrightarrow \quad \text { patient } i \text { is eligible for category } c \text {. }
$$

While in the original problem a patient is indifferent between all units (and therefore all categories as well), in the hypothetical market she has strict preferences between the categories. This "flexibility" in the construction of the hypothetical market is the basis of our main characterization.

For each patient $i \in I$, let $\mathcal{P}_{i}$ be the set of all preferences constructed in this way, and let $\mathcal{P}=\times_{i \in I} \mathcal{P}_{i}$.

Given a preference profile $\succ=\left(\succ_{i}\right)_{i \in I}$, the individual-proposing deferred-acceptance algorithm (DA) produces a matching as follows.

\section{Individual Proposing Deferred Acceptance Algorithm (DA)}

Step 1: Each patient in $I$ applies to her most preferred category among categories for which she is eligible. Suppose that $I_{c}^{1}$ is the set of patients who apply to category $c$. Category $c$ tentatively assigns applicants with the highest priority according to $\pi_{c}$ until all patients in $I_{c}^{1}$ are chosen or all $r_{c}$ units are allocated, whichever comes first, and permanently rejects the rest. If there are no rejections, then stop.

Step k: Each patient who was rejected in Step k-1 applies to her next preferred category among categories for which she is eligible, if such a category exists. Suppose that $I_{c}^{k}$ is the union of the set of patients who were tentatively assigned to category $c$ in Step k-1 and the set of patients who just proposed to category $c$. Category $c$ tentatively assigns patients in $I_{c}^{k}$ with the highest priority according to $\pi_{c}$ until all patients in $I_{c}^{k}$ are chosen or all $r_{c}$ units are allocated, whichever comes first, and permanently rejects the rest. If there are no rejections, then stop.

The algorithm terminates when there are no rejections, at which point all tentative assignments are finalized.

A matching $\mu \in \mathcal{M}$ is called DA-induced if it is the outcome of DA for some preference profile $\succ \in \mathcal{P}$.

We are ready to present our main characterization result:

Theorem 1 A matching is individually rational, non-wasteful, and it respects priorities if, and only if, it is DA-induced.

While our main focus is pandemic rationing, our model so far is general and a reserve system can also be interpreted as an affirmative action system for a wide variety of applications. Since the three axioms can be considered a minimal requirement in this framework, Theorem 1 therefore provides a full characterization of plausible affirmative action policies. 


\subsection{Sequential Reserve Matching}

An interpretation of the DA-induced matchings is helpful to motivate in focusing a subset of these matchings. Recall that the hypothetical two-sided matching market constructed above relies on an artificial preference profile $\left(\succ_{i}\right)_{i \in I}$ of patients over categories. What this corresponds to under the DA algorithm is that any patient $i$ is considered for categories that deem her eligible in sequence, following the ranking of these categories under her artificial preferences $\succ_{i}$. Unless there is a systematic way to construct these preferences, it may be difficult to motivate adopting this methodology for real-life applications. For example, if a patient $i$ is considered first for an unreserved category and then for an essential personnel category, whereas another patient $j$ with similar characteristics is considered for them in the reverse order, it may be difficult to justify this practice. That is, while there are a potentially large class of adequate matchings satisfying our three axioms, not all are necessarily obtained through an intuitive procedure. This may be a challenge especially in the context of medical rationing, since procedural fairness is also an important ethical consideration in this context. Procedural fairness is the main motivation for our focus in a subset of these matchings 17

In many real-life applications of reserve systems, institutions process reserve categories sequentially and allocate units associated with each category one at a time using its categoryspecific priority order. We next formulate matchings obtained in this way and relate them to our characterization in Theorem 1 .

An order of precedence $\triangleright$ is a linear order over the set of categories $\mathcal{C}$. For any two categories $c, c^{\prime} \in \mathcal{C}$,

$$
c \triangleright c^{\prime}
$$

means that category- $c$ units are to be allocated before category- $c^{\prime}$ units. In this case, we say category $c$ has higher precedence than category $c^{\prime}$. Let $\Delta$ be the set of all orders of precedence.

For a given order of precedence $\triangleright \in \Delta$, the induced sequential reserve matching $\varphi_{\triangleright}$, is a matching that is constructed as follows:

Suppose categories are ordered under $\triangleright$ as

$$
c_{1} \triangleright c_{2} \triangleright \ldots \triangleright c_{|\mathcal{C}|}
$$

Matching $\varphi_{\triangleright}$ is found sequentially in $|\mathcal{C}|$ steps:

Step 1: Following their priority order under $\pi_{c_{1}}$, the highest priority $r_{c_{1}}$ category- $c_{1}-$ eligible patients in $I$ are matched with category $c_{1}$. If there are less than $r_{c_{1}}$ eligible patients in $I$ than all of these eligible patients are matched with category $c_{1}$. Let $I_{1}$ be the set of patients matched in Step 1.

Step k: Following their priority order under $\pi_{c_{k}}$, the highest priority $r_{c_{k}}$ category$c_{k^{-}}$-eligible patients in $I \backslash \cup_{k^{\prime}=1}^{k-1} I_{k^{\prime}}$ are matched with category $c_{k}$. If there are less than $r_{c_{k}}$ eligible patients in $I \backslash \cup_{k^{\prime}=1}^{k-1} I_{k^{\prime}}$ then all of these eligible patients are matched with category $c_{k}$. Let $I_{k}$ be the set of patients matched in Step k.

\footnotetext{
${ }^{17}$ Additional discussion of procedural fairness is in Section 4.4
} 
Given an order of precedence $\triangleright \in \Delta$, the induced sequential reserve matching is individually rational, non-wasteful, and it respect priorities. Thus, it is DA-induced by Theorem 1. Indeed it corresponds to a very specific DA-induced matching.

Proposition 1 Fix an order of precedence $\triangleright \in \Delta$. Let the preference profile $\succ^{\triangleright} \in \mathcal{P}$ be such that, for each patient $i \in I$ and pair of categories $c, c^{\prime} \in \mathcal{C}$,

$$
c \succ_{i}^{\triangleright} c^{\prime} \Longleftrightarrow c \triangleright c^{\prime}
$$

Then the sequential reserve matching $\varphi_{\triangleright}$ is DA-induced from the preference profile $\succ^{\triangleright}$.

\subsection{Comparative Statics for Sequential Reserve Matching}

In many real-life applications such as affirmative action in school choice and H1-B visa allocation, there is a baseline priority order $\pi$ of individuals. This priority order may depend on scores on a standardized exam, a random lottery, or arrival time of application. In our main application of pandemic resource allocation, it may depend on SOFA scores described in Section 2.1. This baseline priority order is used to construct the priority order for each of the reserve categories, although each category except one gives preferential treatment to a specific subset of individuals. For example, in our main application these could be essential personnel, Good Samaritans, or people with disabilities. In this section, we focus on this subclass of rationing problems and present an analysis of sequential reserve matching on this class.

To formulate this subclass, we designate a beneficiary group $I_{c} \subseteq I$ for each category $c \in \mathcal{C}$. It is assumed that all patients in its beneficiary group are eligible for a category. That is, for any $c \in \mathcal{C}$ and $i \in I_{c}$,

$$
i \pi_{c} \emptyset \text {. }
$$

There is a category $u \in \mathcal{C}$, called the unreserved category, which has all patients as its beneficiaries and endowed with the same priority order as the baseline priority order. That is,

$$
I_{u}=I \quad \text { and } \quad \pi_{u}=\pi .
$$

Any other category $c \in \mathcal{C} \backslash\{u\}$, referred to as a preferential treatment category, has a more restrictive set $I_{c} \subset I$ of beneficiaries and it is endowed with a priority order $\pi_{c}$ with the following structure: for any pair of patients $i, i^{\prime} \in I$,

$$
\begin{aligned}
i \in I_{c} \quad \text { and } \quad i^{\prime} \in I \backslash I_{c} & \Longrightarrow i \pi_{c} i^{\prime}, \\
i, i^{\prime} \in I_{c} \text { and } i \pi i^{\prime} & \Longrightarrow i \pi_{c} i^{\prime} \text {, and } \\
i, i^{\prime} \in I \backslash I_{c} \text { and } i \pi i^{\prime} & \Longrightarrow i \pi_{c} i^{\prime} .
\end{aligned}
$$

Under $\pi_{c}$, beneficiaries of category $c$ are prioritized over patients who are not, but otherwise their relative priority order is induced by the baseline priority order $\pi$.

While not necessary for practical implementation, in the interest of obtaining sharper results, we make the following assumption in the rest of our analysis:

Assumption 1 Each patient is a beneficiary of at most one preferential treatment category. 
Function $\tau: I \rightarrow(\mathcal{C} \backslash\{u\}) \cup\{\emptyset\}$ identifies which preferential category each patient is a beneficiary of (if any). Here, for any patient $i \in I$,

- $\tau(i)=c$ for some $\mathcal{C} \backslash\{u\}$ means that patient $i$ is a beneficiary of the preferential treatment category $c$ and the unreserved category $u$, whereas

- $\tau(i)=\emptyset$ means that patient $i$ is only a beneficiary of the unreserved category $u$.

Let $I_{g}$, referred to as the set of general-community patients, be the set of patients who are each a beneficiary of the unreserved category only:

$$
I_{g}=\{i \in I: \tau(i)=\emptyset\}=I \backslash \cup_{c \in \mathcal{C} \backslash\{u\}} I_{c} .
$$

We refer to these problems as rationing problems induced by the baseline priority order $\pi$.

In particular two types are such problems have widespread applications.

We say that a priority profile $\left(\pi_{c}\right)_{c \in \mathcal{C}}$ has soft reserves if, for any category $c \in \mathcal{C}$ and any patient $i \in I$,

$$
i \pi_{c} \emptyset \text {. }
$$

Under a soft-reserves rationing problem all individuals are eligible for all categories. This is the case, for example, in our main application of pandemic resource allocation.

We say that a priority profile $\left(\pi_{c}\right)_{c \in \mathcal{C}}$ has hard reserves if, for any preferential treatment category $c \in \mathcal{C} \backslash\{u\}$,

1. $i \pi_{c} \emptyset$ for any of its beneficiaries $i \in I_{c}$, whereas

2. $\emptyset \pi_{c} i$ for any patient $i \in I \backslash I_{c}$ who is not a beneficiary.

Under a hard-reserves rationing problem, only the beneficiaries of a preferred treatment category are eligible for units in this category. This is the case, for example, in H1-B visa allocation in the US.

Allocation rules based on sequential reserve matching are used in a range of practical applications. However, it is important to pay attention to the choice of the order of precedence in these problems, for it has potentially significant distributional implications 18

We obtain the sharpest results on the choice of order of precedence for the case of hard reserves. Therefore in the remainder of this section, we focus on this case. However, since our main application of pandemic rationing is one with soft reserves, we present a version of Theorem 2 in Theorem 5 of Appendix A. Although Theorem 5 is a theoretically weaker result, it is equally relevant for our main application of pandemic rationing. In the same Appendix, we also present two counterexamples showing that the stronger version of the result fails to hold once the hard-reserves assumption is dropped.

Our next result shows that the later a preferential treatment category is processed, the more favorable it is for its beneficiaries at the expense of everyone else.

\footnotetext{
${ }^{18}$ See, for instance, the example in Section 2.3 for an illustration.
} 
Theorem 2 Assuming each patient is a beneficiary of at most one preferential treatment category, consider a hard-reserves rationing problem induced by a baseline priority order $\pi$. Fix a preferential treatment category $c \in \mathcal{C} \backslash\{u\}$, another category $c^{\prime} \in \mathcal{C} \backslash\{c\}$, and a pair of orders of precedence $\triangleright, \triangleright^{\prime} \in \Delta$ such that:

- $c^{\prime} \triangleright c$,

- $c \triangleright^{\prime} c^{\prime}$, and

- for any $\hat{c} \in \mathcal{C}$ and $c^{*} \in \mathcal{C} \backslash\left\{c, c^{\prime}\right\}$,

$$
\hat{c} \triangleright c^{*} \Longleftrightarrow \hat{c} \triangleright^{\prime} c^{*} .
$$

That is, $\triangleright^{\prime}$ is obtained from $\triangleright$ by only changing the order of $c$ with its immediate predecessor $c^{\prime}$. Then,

1. $\varphi_{\triangleright^{\prime}}\left(I_{c}\right) \subseteq \varphi_{\triangleright}\left(I_{c}\right)$ and

2. $\varphi_{\triangleright^{\prime}}\left(I \backslash I_{C}\right) \supseteq \varphi_{\triangleright}\left(I \backslash I_{C}\right)$.

Assuming hard reserves,

- every beneficiary of the preferential treatment category $c$ who is matched by the sequential reserve matching $\varphi_{\triangleright^{\prime}}$ is also matched under the sequential reserve matching $\varphi_{\triangleright}$, and

- every patient who is not a beneficiary of category $c$ and is matched by the sequential reserve matching $\varphi_{\triangleright}$ is also matched under the sequential reserve matching $\varphi_{\triangleright^{\prime}}$.

That is, the later a preferential treatment category is processed the more favorable for its beneficiaries and the less favorable for everyone else.

\subsection{Competing Interests Under Sequential Reserve Matchings}

Theorem 2 motivates a closer look at sequential reserve matchings induced by the following four classes of orders of precedence:

Unreserved Last $\Delta^{u l}$ : For any precedence $\unrhd \in \Delta^{u l}$, each preferential treatment category $c \in \mathcal{C} \backslash\{u\}$ has higher precedence than the unreserved category $u$.

Under elements of this class, the unreserved category is processed after all preferential treatment categories. When there is a single preferential treatment category, the resulting sequential reserve matching, first introduced by Hafalir, Yenmez and Yildirim (2013), is uniquely defined.

Unreserved First $\Delta^{u f}$ : For any precedence $\bar{\nabla} \in \Delta^{u f}$, each preferential treatment category $c \in \mathcal{C} \backslash\{u\}$ has lower precedence than the unreserved category $u$.

PT-c Optimal $\Delta^{c}$ : Fix a preferential treatment category $c \in \mathcal{C} \backslash\{u\}$. For any precedence $\triangleright^{c} \in \Delta^{c}$, the preferential treatment category $c$ has lower precedence than the unreserved category $u$, which itself has lower precedence than any other preferential treatment category $c^{\prime} \in \mathcal{C} \backslash\{c, u\}$. 
PT- $c$ Pessimal $\Delta_{c}$ : Fix a preferential treatment category $c \in \mathcal{C} \backslash\{u\}$. For any precedence $\triangleright_{c} \in \Delta_{c}$, the preferential treatment category $c$ has higher precedence than the unreserved category $u$, which itself has higher precedence than any other preferential treatment category $c^{\prime} \in \mathcal{C} \backslash\{c, u\}$.

We again obtain our sharpest results for hard-reserves rationing problems.

Theorem 3 Assuming each patient is a beneficiary of at most one preferential treatment category, consider a hard-reserves rationing problem induced by a baseline priority order $\pi$. Let $\unrhd \in \Delta^{u l}, \bar{\nabla} \in \Delta^{u f}$, and $\mu \in \mathcal{M}$ be any matching that is individually rational, non-wasteful and that respects priorities. Then,

$$
\varphi_{\bar{\triangleright}}\left(I_{g}\right) \subseteq \mu\left(I_{g}\right) \subseteq \varphi_{\unrhd}\left(I_{g}\right)
$$

That is, of all matchings that satisfy our three axioms, a sequential reserve matching produces

- the best possible outcome under any unreserved last order of precedence, and

- the worst possible outcome under any unreserved first order of precedence

for general-community patients, in a set inclusion sense.

We conclude our formal analysis with a parallel result for beneficiaries of a given preferred treatment category.

Theorem 4 Assuming each patient is a beneficiary of at most one preferential treatment category, consider a hard-reserves rationing problem induced by a baseline priority order $\pi$. Fix a preferential tratment category $c \in \mathcal{C} \backslash\{u\}$. Let $\triangleright_{c} \in \Delta_{c}, \triangleright^{c} \in \Delta^{c}$, and $\mu \in \mathcal{M}$ be any matching that is individually rational, non-wasteful and that respects priorities. Then,

$$
\varphi_{\triangleright_{c}}\left(I_{c}\right) \subseteq \mu\left(I_{c}\right) \subseteq \varphi_{\triangleright^{c}}\left(I_{c}\right)
$$

That is, of all matchings that satisfy our three axioms, a sequential reserve matching produces

- the best possible outcome under any PT-c optimal order of precedence, and

- the worst possible outcome under any PT-c pessimal order of precedence

for the beneficiaries of preferential treatment category $c$ in a set inclusion sense.

\subsection{Related Theoretical Literature}

Our study of reserve systems contributes to literature in matching market design focused on distributional issues. Our main characterization result in Theorem 1 has no antecedent in the literature, and it can be used as a technical tool to analyze other applications of our general model. The comparative statics result presented in Theorem 2 (or Theorem 5 in Appendix) is similar in spirit to Proposition 2 of Dur et al. (2018), but neither result implies the other. In 
contrast to Theorem 2 (or Theorem 5), which analyzes the impact of changing the processing sequence of an entire category as a block in an environment with multiple preferential treatment categories, Proposition 2 in Dur et al. (2018) analyzes the impact of changing the processing sequence of a single unit in an environment with only one preferential treatment category. Theorems 3 and 4 together can be interpreted as a multiple preferential treatment category generalization of the single preferential treatment category result of Theorem 1 in Pathak, ReesJones and Sönmez (2020a). There are also other studies that have examined allocation in the presence constraints such as minimum-guarantee reserves (or lower quotas), upper quotas, and regional quotas. Some of the most related work includes Abdulkadiroğlu (2005), Biro et al. (2010), Kojima (2012), Budish et al. (2013), Hafalir, Yenmez and Yildirim (2013), Westkamp (2013), Ehlers et al. (2014), Echenique and Yenmez (2015), Kamada and Kojima (2015), Kamada and Kojima (2017) Kamada and Kojima (2018), Aygün and Turhan (2016), Aygün and Bo (2016), Bo (2016), Dogan (2016), Kominers and Sönmez (2016), and Fragiadakis and Troyan (2017).

Our paper also introduces the triage protocol design problem into the market design literature. By considering a real-world resource allocation problem, we contribute to the study of formal properties of specific allocation processes in the field and the study of alternative mechanisms. Studies in this vein include those on entry-level labor markets (Roth, 1984; Roth and Peranson, 1999), school choice (Balinski and Sönmez, 1999; Abdulkadiroğlu and Sönmez, 2003 Pathak and Sönmez, 2008, 2013a), spectrum auctions (Milgrom, 2000), kidney exchange (Roth, Sönmez and Ünver, 2004, 2005), internet auctions (Edelman, Ostrovsky and Schwarz, 2007, Varian, 2007), course allocation (Sönmez and Ünver, 2010; Budish, 2011), cadet-branch matching (Sönmez and Switzer, 2013; Sönmez, 2013), assignment of airport arrival slots (Schummer and Vohra, 2013, Schummer and Abizada, 2017), and refugee resettlement (Jones and Teytelboym, 2017; Delacrétaz, Kominers and Teytelboym, 2016; Andersson, 2017).

\section{Additional Considerations for Triage Rationing and Future Directions}

\subsection{Possible Utilization of Excess Units through a Reserve System}

While our analysis pertains to the rationing problem of a single entity, say a hospital or a city, it can be extended to multiple entities. This extension would allow for considerations that can also reduce waste in the system. For example, hospitals in the system can "loan" their unused units to the system, say to a virtual hospital that consists of excess units loaned to the system, and they can earn credit from the system for future use of the units at the virtual hospital when they have a shortage. Hospitals can be incentivized to loan their unused units to the virtual hospital, if their patients receive some priority for some of the units in the virtual hospital. There can be a specific reserve category where priorities may depend on credits earned by the hospitals, while for another category priorities may be determined by clinical criteria only. 


\subsection{Another Application: Rationing of COVID-19 Convalescent Plasma}

Researchers are currently exploring several treatments for patients with COVID-19 including testing existing antiviral drugs and new drug compounds. One potential treatment is known as the convalescent plasma therapy, which involves giving patients an infusion of antibody-rich plasma from patients who have recovered from COVID-19. Preliminary evidence suggests that one unit of convalescent plasma is highly effective against COVID-19 (Duan et al., 2020).

One potential issue with convalescent plasma therapy is a shortage of willing plasma donors. 19 A reserve system with at least two new reserve categories can be designed to encourage donations. One reserve category would give priority to patients of choice for past donors of convalescent plasma. This category is in the same spirit of our Good Samaritan reserve presented in Section 2.4.2. For example, a plasma donor could obtain a number of vouchers, and these would be transferred to patients of their choice. In a second reserve category, a number of units can be reserved for infected patients who promise to donate their plasma to the system once they are healthy and eligible for donation. Since each donor can generate nearly four units of plasma and therefore assist up to four other patients, a donor who benefits from this category could possibly help up to four people. The incentives created with this system could therefore avoid a potential shortage of plasma.

\subsection{Dynamics of Rationing}

For many medical resources such as vaccines, tests, or single-use drug treatment, a patient receives the resource only once. The allocation of other resources is dynamic and subject to periodic review. Currently, there are rules guiding these decisions in existing priority point systems. A reserve system could adhere to exactly the same rules, which may differ between jurisdictions. Some allocation or re-allocation decisions depend on whether units can remain unassigned in expectation of future patient demand, or whether patients can be removed from units they are assigned to due to arrival of higher-priority patients. For instance, one view stated by the 2015 New York State task force is:

... removing a ventilator from a patient who worsens or does not improve so that another patient with a strong likelihood of survival may have an opportunity for treatment helps support the goal of saving the greatest number of lives in an influenza pandemic where there are a limited number of ventilators.

Another view might suggest that a patient cannot be removed once assigned. Still, another view calls for periodic clinical assessments at pre-specified time intervals and allows for changing the assignments after this review. In this situation, the reserve system could be deployed for an updated set of patient types and total supply of the medical resource (which also may change over time).

There is no consensus on the correct approach to solve these difficult questions. However, rationing guidelines give specific directions to resolve them and the implementation of existing

\footnotetext{
${ }^{19}$ Al-Jazeera (2020) describes efforts to obtain plasma donations from recovered patients. Institutions including the Mayo Clinic and the American Red Cross have already established programs accepting plasma donations.
} 
priority point systems are expected to comply with these rules. Reserve systems can also comply with the same rules if desired. After all, both a priority point system and a reserve system can be interpreted as accounting systems for applying certain balances of ethical values in managing scarce medical resources.

\subsection{Reserve Systems Compared to Constrained Optimization}

We briefly contrast a reserve system with an alternative approach that tries to accommodate multiple ethical values using constrained optimization 20 In a constrained optimization approach, there is an objective function and there are constraints. Perhaps certain ethical values can be aggregated into an objective function, while others can be formulated as constraints. For example, suppose the two ethical goals are to maximize expected health outcomes and non-discrimination. It is a non-trivial task to aggregate these two goals into a single objective function. This is indeed not very different than some of the challenges faced in multi-principle point systems. But suppose we are indeed able to find a representative objective function. Further let us assume that the remaining ethical considerations can be mathematically formulated as constraints. Assuming this maximum can be computed and multiplicities do not cause any issue concerning procedural fairness, we still see several advantages of using a reserve system.

First, a reserve system allows for non-consequentialist ethical goals such as those related to procedural fairness. Clarity on the process by which allocations are determined is an important part of many rationing guidelines. Michigan's standards, for example, state that (Michigan, 2012 , page 21)

procedural justice requires that fair and clear processes be used to make allocation decisions...

Furthermore, disability rights groups reject any consideration of probability or length of survival, let alone maximization of these aims (DREDF, 2020). Ne'eman $(2020)$ argues that the principle of non-discrimination should be seen as an ends by itself. Because a constrained optimization approach optimizes over allocations, and not the process by which allocations are chosen, its relationship to procedural aims is less obvious.

Second, reserve systems have straightforward parameters that can be directly linked to ethical principles. Since community involvement is an integral part of designing rationing guidelines, easy-to-interpret parameters are desirable. A Johns Hopkins study on community perceptions states (Biddison et al., 2013):

Both groups felt strongly that no single principle could adequately balance the competing aims and values triggered by allocation decisions. Some felt that a combination of principles should be used.

Suppose that a task force proposes a reserve category for essential personnel for a fraction resources, then because any preferential treatment has to be based on well-established ethical criteria, it is clear that the guidelines incorporate the principles of reciprocity and instrumental

\footnotetext{
${ }^{20}$ We are grateful to Eric Budish for suggesting this section.
} 
value. And public acceptance is an essential part of any rationing guideline. For example, New York's guidelines emphasize transparency and state that the "process of developing a clinical ventilator allocation protocol is open to feedback and revision, which helps promote public trust." (Zucker et al., 2015, page 5).

Third, we believe formulating competing objectives within constrained optimization approaches may be hard to do with potentially conflicting views and opinions. Assuming each group can even formulate a clear objective function, aggregation across different objective functions into a single objective function requires placing competing aims on a common scale, which may be unnatural ${ }^{21}$ In a reserve system, each ethical principle or a balance between multiple principles can be linked to a reserve category, eliminating the technical need to formulate a single objective.

Fourth, it is reassuring that reserve systems are often used in applications that involve community involvement. For example, to accommodate a higher degree of efficiency in the deceased donor kidney allocation system, OPTN introduced in 2014 a reserve category which gives preferential treatment to patients with the highest expected benefit for $20 \%$ of the highest quality deceased-donor kidneys after widespread community outreach. Similarly, following debates between the pro-neighborhood and pro-choice factions, Boston's school assignment system established a reserve where half of each school's seats prioritize applicants from the walk zone. Likewise, reserves were developed as part of India's affirmative action system after more than a decade of community involvement summarized in the 1979 Mandal Commission Report and formulated in the landmark 1992 Indra Sahwney Supreme Court case. These reserve-system precedents suggest that stakeholders might prefer the natural interpretation of reserve categories.

Finally, an alternative allocation mechanism may be more likely to be adopted if it is not a substantial change from the existing rules. Currently, the two leading mechanisms, a single principle SOFA-score based system and White et al. (2009)'s multi-principle system, are both priority point systems. Since a reserve system can easily build on these priority point systems, overcoming their difficulties without fundamental changes, it may receive more widespread acceptance.

\section{Conclusion}

Because of the anticipated and ongoing shortage of key medical resources during the COVID-19 pandemic, several leaders in the medical ethics community have made important recommendations regarding medical rationing. These recommendations reflect compromises between several ethical principles - maximizing lives, maximizing life-years, life-cycle considerations, instrumental values, reciprocity, protecting to the sickest, and non-exclusion. The priority point system, which aggregates all ethical dimensions into a single score, has become the norm. A reserve system offers additional flexibility and can simultaneously balance competing objectives in ways that a priority point system cannot.

We have shown that reserve systems can remedy challenges associated with prioritizing

\footnotetext{
${ }^{21}$ There is an entire subfield of philosophy about commensurable and incommensurable objectives. See, e.g., Hsieh (2016).
} 
frontline health workers, an issue that has befuddled the medical ethics community. Emanuel et al. (2020) and White and Lo (2020) argue that these essential personnel should be prioritized, but several state standards express the concern that if frontline health workers are given priority, they could exhaust total supply and leave no resources for patients from the general community. By setting aside a fraction of vital medical resources for essential personnel as in a reserve system, not only can these concerns be overcome, but the ethical principles of reciprocity and instrumental valuation can also be reflected in the rationing system.

The theory of reserve systems we develop is based on three simple criteria of non-wastefulness, individual rationality, and respect for priority. Non-wastefulness means no medical resource is unused while there are patients who are in need. Individual rationality means if there are exclusion criteria for certain reserve categories, they have to be respected. For life-saving medical resources, it is natural to rule out category-specific exclusion criteria, so we expect this criteria to hold automatically for triage protocol design. Respect for priority means that allocation of units in a given category has to follow the priority order of patients in that category. To the extent the priority order of patients represents the ethical principles guiding the allocation, this is also a natural principle. It is important to emphasize that these are the same principles that are embraced by the existing triage systems. What differs in our analysis is that we allow for different ethical principles to guide allocation decisions across different units.

In our formal analysis, we characterize the entire class of reservation policies that satisfy three minimal principles though implementation of the deferred-acceptance algorithm. As such, we also provide a full characterization of affirmative action policies. In the context of triage protocol design, one of the main insights for implementation of reserve systems is that processing a reserve category earlier is detrimental to its beneficiaries.

While our analysis has focused on triage protocol design, our results can be directly applied to several other resource allocation problems with reserves. Within medicine, specific resources like vaccines and plasma therapy may require additional considerations which differ from ventilators. Outside of medicine, our results are relevant for immigration visa allocation in the United States (Pathak, Rees-Jones and Sönmez, 2020a), affirmative action in school choice systems in Boston, Chicago, New York City and Chile (Dur et al., 2018; Dur, Pathak and Sönmez, 2019; Correa et al. 2019), affirmative action for public school and government positions in India (Aygün and Turhan, 2017; Baswana et al., 2018; Sönmez and Yenmez, 2019a b), and diversity plans for college admissions in Brazil (Aygün and Bo, 2016). We leave explorations of these connections to future research.

There are three main design parameters in a reserve system: the reserve categories and their sizes, the reserve category processing order, and the priority order in each reserve category. Choices about these dimensions still require decisions about ethical principles and their appropriate weight. However, our theory allows for greater separation of ethical principles from their implementation, thereby allowing medical ethicists, state task forces, and other stakeholders additional flexibility to integrate multiple ethical concerns. When these decisions are made, we believe it is important to precisely define which ethical principles govern these choices and make these details transparent to all stakeholders.

We hope that the triage rationing protocol we have analyzed will only be necessary in 
exceptional circumstances during the current pandemic and for future ones. However, even if rationing guidelines are never applied, their mere existence reflects a statement of values. Several aspects of the design, including those related to essential personnel, adults and children, and the rights of the disabled, run the risk of upsetting social harmony. For example, Fink (2020) describes that "at the end you have got a society at war with itself. Some people are going to be told they don't matter enough." We have shown that a reserve system provides policy makers with an additional tool to navigate these complex challenges. Although confronting scarcity in life-and-death situations is dire, we also hope some of our ideas, such as creating a Good Samaritan category, hold the potential to use the current crisis to serve other longstanding medical problems not related to COVID-19. 


\section{Appendix A Comparative Statics without the Hard-Reserves Assumption}

Since the hard-reserves assumption fails to hold in our main application of pandemic rationing, we present in this Appendix a variant of Theorem 2 in the absence of this assumption. There are two consequences of dropping the hard-reserve assumption:

1. The first implication is only guaranteed to hold if there are no more than four categories ${ }^{22}$

2. The second implication of the theorem no longer holds: in the absence of the hard-reserves assumption, processing a category $c$ later in the sequence is not necessarily detrimental for individuals who are not beneficiaries of category $c$.

Theorem 5 Suppose $|\mathcal{C}| \leq 4$. Assuming each patient is a beneficiary of at most one preferential treatment category, consider a rationing problem induced by a baseline priority order $\pi$. Fix a preferential treatment category $c \in \mathcal{C} \backslash\{u\}$, another category $c^{\prime} \in \mathcal{C} \backslash\{c\}$, and a pair of orders of precedence $\triangleright, \triangleright^{\prime} \in \Delta$ such that:

- $c^{\prime} \triangleright c$,

- $c \triangleright^{\prime} c^{\prime}$, and

- for any $\hat{c} \in \mathcal{C}$ and $c^{*} \in \mathcal{C} \backslash\left\{c, c^{\prime}\right\}$,

$$
\hat{c} \triangleright c^{*} \Longleftrightarrow \hat{c} \triangleright^{\prime} c^{*} .
$$

That is, $\triangleright^{\prime}$ is obtained from $\triangleright$ by only changing the order of $c$ with its immediate predecessor $c^{\prime}$. Then,

$$
\varphi_{\triangleright^{\prime}}\left(I_{c}\right) \subseteq \varphi_{\triangleright}\left(I_{c}\right)
$$

This result shows that as long as there are not more than three preferential treatment categories in addition to the unreserved category, every beneficiary of the preferential treatment category $c$ who is matched by the sequential reserve matching $\varphi_{\triangleright^{\prime}}$ is matched also under the sequential reserve matching $\varphi_{\triangleright}$.

On the other hand, the following example shows that when the number of categories is six or more, the first conclusion of Theorem 2 no longer holds.

Example 1 Suppose there are $q=6$ medical units to be allocated in total. There are 6 categories: the unreserved category $u$ and 5 preferential treatment categories $c, c^{\prime}, c^{*}, \hat{c}, \tilde{c}$ and each category has a single unit capacity.

Suppose there are 7 patients. All patients are beneficiaries of the unreserved category $u$ :

$$
I_{u}=\left\{i_{1}, i_{2}, i_{3}, i_{4}, i_{5}, i_{6}, i_{7}\right\}
$$

\footnotetext{
${ }^{22}$ We conjecture that this implication holds when there are five categories as well. However, as shown in Example 1] the result fails to hold when there are six or more categories. Therefore the proof of Theorem 5 is through a brute-force analysis of all cases. Since the practically relevant cases will likely be already covered with an upper bound of four categories, we leave the case of five categories as an open question.
} 
The beneficiaries of preferential treatment categories $c, c^{*}$, and $\tilde{c}$ are given as

$$
I_{c}=\left\{i_{1}, i_{3}, i_{6}\right\}, \quad I_{c^{*}}=\left\{i_{2}, i_{5}\right\}, \quad I_{\tilde{c}}=\left\{i_{4}, i_{7}\right\},
$$

while there are no beneficiaries of preferential treatment categories $c^{\prime}$ and $\hat{c}: I_{c^{\prime}}=\emptyset$ and $I_{\hat{c}}=\emptyset$. There are also no general-community patients: $I_{g}=\emptyset$. Suppose $\pi$, the baseline priority order of patients, is given as

$$
i_{1} \pi i_{2} \pi i_{3} \pi i_{4} \pi i_{5} \pi i_{6} \pi i_{7} .
$$

Also assume that all patients are eligible for all preferential treatment categories besides the unreserved category $u$.

We consider a sequential reserve matching based on the following order of precedence $\triangleright$ :

$$
c^{\prime} \triangleright c \triangleright c^{*} \triangleright \hat{c} \triangleright \tilde{c} \triangleright u \text {. }
$$

This sequential reserve matching matches $\varphi_{\triangleright}(I)=\left\{i_{1}, i_{3}, i_{2}, i_{4}, i_{7}, i_{5}\right\}$ in the order agents are written in this set. In this case,

$$
\varphi_{\triangleright}\left(I_{c}\right)=\left\{i_{1}, i_{3}\right\}
$$

is the set of matched category-c beneficiaries.

We compare this outcome with the sequential reserve matching under the order of precedence $\triangleright^{\prime}$ that switches the order of $c$ and $c^{\prime}$, and otherwise, leaves the order of other categories the same as under $\triangleright$ :

$$
c \triangleright^{\prime} c^{\prime} \triangleright^{\prime} c^{*} \triangleright^{\prime} \hat{c} \triangleright^{\prime} \tilde{c} \triangleright^{\prime} u \text {. }
$$

This sequential reserve matching matches $\varphi_{\triangleright^{\prime}}(I)=\left\{i_{1}, i_{2}, i_{5}, i_{3}, i_{4}, i_{6}\right\}$ in the order patients are written in this set. In this case,

$$
\varphi_{\triangleright^{\prime}}\left(I_{c}\right)=\left\{i_{1}, i_{3}, i_{6}\right\}
$$

is the set of matched category-c beneficiaries.

Thus,

$$
\varphi_{\triangleright^{\prime}}\left(I_{C}\right) \supsetneq \varphi_{\triangleright}\left(I_{C}\right)
$$

although $c$ is is ordered earlier under $\triangleright^{\prime}$ than under $\triangleright$.

Finally the following example shows that in the absence of the hard-reserves assumption, the second conclusion fails even with only two preferential-treatment categories.

Example 2 There is an unreserved category $u$ and two preferential treatment categories $c, c^{\prime}$. There is one medical unit reserved for each category, and the beneficiary groups are $I_{u}=$ $\left\{i_{1}, i_{2}, i_{3}, i_{4}\right\}, I_{c}=\left\{i_{1}\right\}$ and $I_{c^{\prime}}=\left\{i_{2}, i_{4}\right\}$. All patients are eligible for all categories. Let $\triangleright$, $\triangleright^{\prime}$ be two precedence orders such that

$$
u \triangleright c^{\prime} \triangleright c \quad \text { and } \quad u \triangleright^{\prime} c \triangleright^{\prime} c^{\prime} \text {. }
$$

Finally, the baseline priority order $\pi$ is given as

$$
i_{1} \pi i_{2} \pi i_{3} \pi i_{4}
$$


Under the sequential reserve matching $\varphi_{\triangleright}$ the set of patients who are matched is $\left\{i_{1}, i_{2}, i_{3}\right\}$, and under the sequential reserve matching $\varphi_{\triangleright^{\prime}}$ the set of patients who are matched is $\left\{i_{1}, i_{2}, i_{4}\right\}$. Therefore, when the order of precedence is change from $\triangleright$ to $\triangleright^{\prime}$, a move that is (weakly) detrimental to beneficiaries of category $c$ by Theorem 5, patient $i_{3}$, who is not a beneficiary of this category, is made worse off. This shows a change that potentially hurts beneficiaries of one category may hurt other patients as well.

\section{Appendix B Proofs}

\section{Proof of Theorem 1.}

Sufficiency: We first prove that any DA-induced matching is individually rational, nonwasteful and it respects priorities. Let $\succ \in \mathcal{P}$ be a preference profile of patients over categories and $\emptyset$. Suppose $\mu \in \mathcal{M}$ is DA-induced from this preference profile.

Individual rationality: Suppose that $\mu(i)=c$ for some $c \in \mathcal{C}$. Then $i$ must apply to $c$ in a step of the DA algorithm, and hence, $c \succ_{i} \emptyset$. By construction of $\succ_{i}$, this means $i \pi_{c} \emptyset$. Therefore, matching $\mu$ is individually rational.

Non-wastefulness: Suppose that $i \pi_{c} \emptyset$ and $\mu(i)=\emptyset$ for some category $c \in \mathcal{C}$ and patient $i \in I$. By construction of $\succ_{i}, c \succ_{i} \emptyset$ because she is eligible for $c$. As agent $i$ remains unmatched in $\mu$, she applies to $c$ in some step of the DA algorithm. However, $c$ rejects $i$ at this or a later step. This means, $c$ should have been holding at least $r_{c}$ offers from eligible students at this step. From this step on, $c$ always holds $r_{c}$ offers and eventually all of its units are assigned: $\left|\mu^{-1}(c)\right|=r_{c}$. Hence, matching $\mu$ is non-wasteful.

Respecting priorities: Suppose that $\mu(i)=c$ and $\mu\left(i^{\prime}\right)=\emptyset$ for two patients $i, i^{\prime} \in I$ and a category $c \in \mathcal{C}$. For every category $c^{\prime} \in \mathcal{C}, \pi_{c^{\prime}}$ is used to choose eligible patients at every step of the DA algorithm. Therefore, $\mu(i)=c$ implies $i \pi_{c} \emptyset$. Since $\mu\left(i^{\prime}\right)=\emptyset$, then it must be either because $\emptyset \pi_{c} i^{\prime}$ or because $i \pi_{c} i^{\prime}$. In the first case, we get $i \pi_{c} i^{\prime}$ as well because $\pi_{c}$ is transitive. Therefore, matching $\mu$ respects priorities.

Necessity: We now prove that any matching $\mu \in \mathcal{M}$ with the three stated properties is DAinduced from some preference profile. We construct a candidate preference profile $\succ \in \mathcal{P}$ as follows:

- Consider a patient $i \in \mu^{-1}(c)$ where $c \in \mathcal{C}$. Since $\mu$ is individually rational, $i$ must be eligible for category $c$. Let $i$ rank category $c$ first in $\succ_{i}$. The rest of the ranking in $\succ_{i}$ is arbitrary as long as all eligible categories are ranked above the empty set.

- Consider an unmatched patient $i \in \mu^{-1}(\emptyset)$. Let $i$ rank categories in any order in $\succ_{i}$ such that only eligible categories are ranked above the empty set.

We nest show that $\mu$ is DA-induced from preference profile $\succ$. In the induced DA algorithm under $\succ$, for every category $c^{\prime} \in \mathcal{C}$, patients in $\mu^{-1}\left(c^{\prime}\right)$ apply to category $c^{\prime}$ first. Every unmatched patient $j \in \mu^{-1}(\emptyset)$ applies to her first-ranked eligible category according to $\succ_{j}$, if there is any. Suppose $c \in \mathcal{C}$ is this category. Since $\mu$ respects priorities, $j$ has a lower priority than any patient 
in $\mu^{-1}(c)$, who also applied to $c$ in Step 1. Furthermore, since $\mu$ is non-wasteful, $\left|\mu^{-1}(c)\right|=r_{c}$ (as there are unmatched eligible patients for this category, for example $j$ ). Therefore, all unmatched patients in $\mu$ are rejected at the first step of the DA algorithm. Moreover, for every category $c^{\prime} \in \mathcal{C}$, all patients in $\mu^{-1}\left(c^{\prime}\right)$ are tentatively accepted by category $c^{\prime}$ at the end of Step 1.

Each unmatched patient in $j \in \mu^{-1}(\emptyset)$ continues to apply according to $\succ_{j}$ to the other categories at which she is eligible. Since $\mu$ respects priorities and is non-wasteful, she is rejected from all categories for which she is eligible one at a time: that is because each of these categories $c \in \mathcal{C}$ continues to tentatively hold patients $\mu^{-1}(c)$ from step 1 who have all higher priority than $j$ according to $\pi_{c}$, as $\mu$ respects priorities. Moreover, by non-wastefulness of $\mu,\left|\mu^{-1}(c)\right|=r_{c}$, as there are unmatched eligible patients (for example $j$ ) under $\mu$.

As a result when the algorithm stops, the outcome is such that, for each category $c^{\prime} \in \mathcal{C}$, all patients in $\mu^{-1}\left(c^{\prime}\right)$ are matched with $c^{\prime}$. Moreover, every patient in $\mu^{-1}(\emptyset)$ remains unmatched at the end. Therefore, $\mu$ is DA-induced from the constructed patient preferences $\succ$.

Proof of Proposition 1. Let $\triangleright \in \Delta$ be a precedence order and $\varphi_{\triangleright}$ be the associated sequential reserve matching. We show that $\varphi_{\triangleright}$ is DA-induced from preference profile $\succ^{\triangleright}=$ $\left(\succ_{i}^{\triangleright}\right)_{i \in I}$.

For each patient $i \in I$, consider another strict preference relation $\succ_{i}^{\prime}$ such that all categories are ranked above the empty set and, furthermore, for any $c, c^{\prime} \in \mathcal{C}$,

$$
c \succ_{i}^{\prime} c^{\prime} \Longleftrightarrow c \triangleright c^{\prime}
$$

Note that the relative ranking of two categories for which $i$ is eligible is the same between $\succ_{i}^{\triangleright}$ and $\succ_{i}^{\prime}$.

We use an equivalent version of the DA algorithm as the one given in the text. Consider a Step $k$ : Each patient $i$ who is not tentatively accepted currently by a category applies to the best category that has not rejected her yet according to $\succ_{i}^{\prime}$. Suppose that $I_{c}^{k}$ is the union of the set of patients who were tentatively assigned to category $c$ in Step $k-1$ and the set of patients who just proposed to category $c$. Category $c$ tentatively assigns eligible patients in $I_{c}^{k}$ with the highest priority according to $\pi_{c}$ until all patients in $I_{c}^{k}$ are chosen or all $r_{c}$ units are allocated, whichever comes first, and permanently rejects the rest.

Since for any category $c \in \mathcal{C}$ and any patient $i \in I$ who is ineligible for category $c, \emptyset \pi_{c} c$, the outcome of the DA algorithm when the preference profile is $\succ^{\triangleright}$ and $\succ^{\prime}=\left(\succ_{i}^{\prime}\right)_{i \in I}$ are the same.

Furthermore, when the preference profile is $\succ^{\prime}$, the DA algorithm works exactly like the sequential reserve procedure that is used to construct $\varphi_{\triangleright}$. We show this by induction. Suppose $\triangleright$ orders categories as $c_{1} \triangleright c_{2} \triangleright \ldots \triangleright c_{|\mathcal{C}|}$. As the inductive assumption, for $k>0$, suppose for categories $c_{1}, \ldots, c_{k-1}$, the tentative matches at the end of Step $k-1$ and final matches at the end under the DA algorithm from $\succ^{\prime}$ are identical to their matches in sequential reserve matching $\varphi_{\triangleright}$.

We next consider Step $k$ of the DA algorithm from $\succ^{\prime}$. Only patients who are rejected from category $c_{k-1}$ apply in Step $k$ of the DA algorithm and they all apply to category $c_{k}$. Then $c_{k}$ uses its priority order $\pi_{c_{k}}$ to tentatively accept the $r_{c_{k}}$ highest-priority eligible applicants (and if there are less than $r_{c_{k}}$ eligible applicants, all eligible applicants), and rejects the rest. Observe 
that since every patient who is not tentatively accepted by a category $c_{1}, \ldots, c_{k-1}$ applied to this category in Step $k$, none of these patients will ever apply to it again; and by the inductive assumption no patient who is tentatively accepted in categories $c_{1}, \ldots, c_{k-1}$ will ever be rejected, and thus, they will never apply to $c_{k}$, either. Thus, the tentative acceptances by $c_{k}$ will become permanent at the end of the DA algorithm. Thus, this step is identical to Step $k$ of the sequential reserve procedure under precedence order $\triangleright$ and the same patients are matched to category $c_{k}$ in $\varphi_{\triangleright}$. This ends the induction.

Therefore, we conclude that $\varphi_{\triangleright}$ is DA-induced from patient preference profile $\succ^{\triangleright}$.

Proof of Theorem 2, The sequential reserve matchings $\varphi_{\triangleright}$ and $\varphi_{\triangleright^{\prime}}$ match the same patients to the categories with higher precedence than $c$ and $c^{\prime}$ under both $\triangleright$ and $\triangleright^{\prime}$. Let $J \subseteq I$ refer to the set of patients who are available when $c^{\prime}$ is about to be processed under $\triangleright^{\prime}$ (or equivalently when $c$ is about to be processed under $\triangleright$ ) in the sequential reserve matching procedure.

Two cases are possible: For the unreserved category $u$, either $c^{\prime} \neq u$ or $c^{\prime}=u$. We consider these two separately.

Case 1. $c^{\prime} \neq u$ : Then $c^{\prime}$ is a preferential treatment category as well. Since the problem is hardreserves, $\varphi_{\triangleright}^{-1}\left(c^{*}\right) \subseteq J_{c^{*}}$ for each $c^{*} \in\left\{c, c^{\prime}\right\}$ and $\varphi_{\triangleright^{\prime}}^{-1}\left(c^{*}\right) \subseteq J_{c^{*}}$ for each $c^{*} \in\left\{c, c^{\prime}\right\}$. Then the order of $c^{\prime}$ and $c$ do not matter and we have $\varphi_{\triangleright}^{-1}\left(c^{*}\right)=\varphi_{\triangleright^{\prime}}^{-1}\left(c^{*}\right)$ for each $c^{*} \in\left\{c, c^{\prime}\right\}$. Hence under both $\triangleright$ and $\triangleright^{\prime}$, after $c$ and $c^{\prime}$ are processed the same set of patients remain unmatched. Since the order of the subsequent categories are the same, both matchings $\varphi_{\triangleright^{\prime}}$ and $\varphi_{\triangleright}$ match the same set of patients subsequently to the same categories. Since $\varphi_{\triangleright^{\prime}}$ and $\varphi_{\triangleright}$ also match the same patients among $I \backslash J$ to the same categories prior to $c$ and $c^{\prime}$, we obtain $\varphi_{\triangleright^{\prime}}=\varphi_{\triangleright}$, which also implies

$$
\varphi_{\triangleright^{\prime}}\left(I_{c}\right)=\varphi_{\triangleright}\left(I_{c}\right) \quad \text { and } \quad \varphi_{\triangleright^{\prime}}\left(I \backslash I_{c}\right)=\varphi_{\triangleright}\left(I \backslash I_{c}\right),
$$

proving the theorem for Case 1.

Case 2. $c^{\prime}=u$ : Then $u \triangleright c$ while $c \triangleright^{\prime} u$ and they are consecutively ordered. We are choosing patients among $J_{c}$, with respect to the same priority order $\pi$ to fill either $u$ and $c$ implying that weakly a larger set of category $c$ beneficiaries are matched to $\{u, c\}$ under $\triangleright$ with respect to $\triangleright^{\prime}$ :

$$
\varphi_{\triangleright^{\prime}}^{-1}\left(\left\{c^{\prime}, u\right\}\right) \cap J_{c} \subseteq \varphi_{\triangleright}^{-1}\left(\left\{c^{\prime}, u\right\}\right) \cap J_{c} .
$$

This also implies

$$
\varphi_{\triangleright^{\prime}}^{-1}(u) \cap\left(J \backslash J_{c}\right) \supseteq \varphi_{\triangleright}^{-1}(u) \cap\left(J \backslash J_{c}\right) .
$$

Thus, as any general-community category patient in $I_{g}$ can is only eligible for the unreserved category $u$ because of the hard-reserves feature, by Relationship (2)

$$
\varphi_{\triangleright^{\prime}}\left(I_{g}\right) \supseteq \varphi_{\triangleright}\left(I_{g}\right) .
$$

Recall that $\varphi_{\triangleright^{\prime}}$ and $\varphi_{\triangleright}$ match the same patients to the categories ordered before $c$ under $\triangleright^{\prime}$ and before $u$ under $\triangleright$. There are three cases for the patients in $I \backslash I_{g}$ : 
- Since category-c beneficiaries are not eligible for any other category ordered after $c$ and $u$ in a hard-reserves problem, then no category- $c$ beneficiary is matched after this step. By relationship (1), we obtain

$$
\varphi_{\triangleright^{\prime}}\left(I_{c}\right) \subseteq \varphi_{\triangleright}\left(I_{c}\right)
$$

- For any preferential treatment category $c^{*} \notin\{c, u\}$ ordered before $c$ under $\triangleright^{\prime}$ and before $u$ under $\triangleright$, by relationship (2), we obtain

$$
\varphi_{\triangleright^{\prime}}\left(I_{c^{*}}\right) \supseteq \varphi_{\triangleright}\left(I_{c^{*}}\right) .
$$

- For any preferential treatment category $c^{*} \notin\{c, u\}$ ordered after $u$ under $\triangleright^{\prime}$ and after $c$ under $\triangleright$, since we are choosing patients among $I_{c^{*}}$, with respect to the same priority order $\pi$ to fill either $u$ and $c^{*}$ and these patients are not eligible for any other preferential-treatment category because of the hard-reserves feature, by relationship (2)

$$
\varphi_{\triangleright^{\prime}}\left(I_{c^{*}}\right) \supseteq \varphi_{\triangleright}\left(I_{c^{*}}\right)
$$

The last four displayed relationships prove the theorem for Case 2.

These complete the proof of the theorem.

Proof of Theorem 3. Let $\bar{\nabla} \in \Delta^{u f}$ be an unreserved-first order of precedence and $\unrhd \in \Delta^{u l}$ be an unreserved-last order of precedence given a hard-reserves problem induced by the baseline priority order $\pi$. Note that in a hard-reserves problem, general-community patients are only eligible for the unreserved category. Let $\mu \in \mathcal{M}$ be a matching that is individually rational, non-wasteful, and that respects priorities. For a set of patients $\tilde{I} \subseteq I$ and a patient $i \in \tilde{I}$ who is eligible at a category $c \in \mathcal{C}$, let $\operatorname{rank}\left(i ; \tilde{I}, \pi_{c}\right)$ denote the rank of $i$ among patients in $\tilde{I}$ according to $\pi_{c}$. For example, the patient with the highest priority has rank one.

We prove the result in two separate claims.

Claim 1: $\varphi_{\bar{\triangleright}}\left(I_{g}\right) \subseteq \mu\left(I_{g}\right)$.

Proof. Note that $\varphi_{\triangleright}$ is a sequential reserve matching when the unreserved category is processed first. Thus, $\varphi_{\bar{\triangleright}}^{-1}(u)$ is the set of $r_{u}$-highest priority patients according to $\pi$, which implies that $i \in \varphi_{\bar{\triangleright}}\left(I_{g}\right)$ if, and only if, $i$ is among the $r_{u}$-highest priority patients according to $\pi$ in $I$. As a result, in $\mu$, which respects priorities and non-wasteful, $i \in \varphi_{\bar{\triangleright}}\left(I_{g}\right)$ is matched with category $u$, as this is the only eligible category for general-community patients. This shows that $\varphi_{\bar{\triangleright}}\left(I_{g}\right) \subseteq \mu\left(I_{g}\right)$.

Claim 2: $\mu\left(I_{g}\right) \subseteq \varphi_{\unrhd}\left(I_{g}\right)$.

Proof. For each preferential treatment category $c \in \mathcal{C} \backslash\{u\},\left|\varphi_{\unrhd}^{-1}(c)\right| \geq\left|\mu^{-1}(c)\right|$ and every patient in $\varphi_{\unrhd}^{-1}(c) \backslash \mu^{-1}(c)$ has a strictly higher priority according to $\pi$ than every patient in $\mu^{-1}(c) \backslash \varphi_{\unrhd}^{-1}(c)$ since $\varphi_{\unrhd}$ is generated by unreserved-last precedence order $\unrhd$. Furthermore, as $\mu$ is DA-induced by Theorem 1 .

$$
\mu^{-1}(u)=\left\{i \in I \backslash \cup_{c \in \mathcal{C} \backslash\{u\}} \mu^{-1}(c): \operatorname{rank}\left(i ; I \backslash \cup_{c \in \mathcal{C} \backslash\{u\}} \mu^{-1}(c), \pi\right) \leq r_{u}\right\} .
$$


As the unreserved category $u$ is processed last in finding $\varphi_{\unrhd}$ and this is a hard-reserves problem,

$$
\varphi_{\unrhd}^{-1}(u)=\left\{i \in I \backslash \cup_{c \in \mathcal{C} \backslash\{u\}} \varphi_{\unrhd}^{-1}(c): \operatorname{rank}\left(i ; I \backslash \cup_{c \in \mathcal{C} \backslash\{u\}} \varphi_{\unrhd}^{-1}(c), \pi\right) \leq r_{u}\right\} .
$$

Take $i \in \mu\left(I_{g}\right)$. Then $i \in \mu^{-1}(u)$, which implies that

$$
\operatorname{rank}\left(i ; I \backslash \cup_{c \in \mathcal{C} \backslash\{u\}} \mu^{-1}(c), \pi\right) \leq r_{u} .
$$

Since, for each preferential treatment category $c \in \mathcal{C} \backslash\{u\},\left|\varphi_{\unrhd}^{-1}(c)\right| \geq\left|\mu^{-1}(c)\right|$ and every patient in $\varphi_{\unrhd}^{-1}(c) \backslash \mu^{-1}(c)$ has a strictly higher priority according to $\pi$ than every patient in $\mu^{-1}(c) \backslash \varphi_{\unrhd}^{-1}(c)$, the above displayed inequality implies

$$
\operatorname{rank}\left(i ; I \backslash \cup_{c \in \mathcal{C} \backslash\{u\}} \varphi_{\unrhd}^{-1}(c), \pi\right) \leq r_{u} .
$$

Therefore, $i \in \varphi_{\unrhd}^{-1}(u)$, which is equivalent to $i \in \varphi_{\unrhd}\left(I_{g}\right)$.

We conclude that $\mu\left(I_{g}\right) \subseteq \varphi_{\unrhd}\left(I_{g}\right)$.

Proof of Theorem 4. Consider a hard-reserves problem induced by a baseline priority order $\pi$. Fix a preferential treatment category $c \in \mathcal{C} \backslash\{u\}$, a PT-c pessimal order of precedence $\triangleright_{c} \in \Delta_{c}$, and a PT-c optimal order of precedence $\triangleright^{c} \in \Delta^{c}$. Also let $\mu \in \mathcal{M}$ be an individually rational and non-wasteful matching that also respects priorities.

For a set of patients $\tilde{I} \subseteq I$ and patient $i \in \tilde{I}$ who is eligible at a category $\tilde{c} \in \mathcal{C}$, let $\operatorname{rank}\left(i ; \tilde{I}, \pi_{\tilde{c}}\right)$ denote the rank of $i$ among patients in $\tilde{I}$ according to $\pi_{\tilde{c}}$.

We prove the result in two separate claims.

Claim 1: $\varphi_{\triangleright_{c}}\left(I_{c}\right) \subseteq \mu\left(I_{c}\right)$.

Proof. Note that $\varphi_{\triangleright_{c}}$ is a sequential reserve matching generated by the PT-c pessimal precedence order $\triangleright_{c}$ that orders $c$ before $u$ and orders $u$ before all other preferential treatment categories. Therefore,

$$
\varphi_{\triangleright_{c}}\left(I_{c}\right)=\widehat{I}_{c} \cup \widetilde{I}_{c}
$$

where

$$
\widehat{I}_{c}=\varphi_{\triangleright_{c}}^{-1}(c)=\left\{i \in I_{c}: \operatorname{rank}\left(i ; I_{c}, \pi\right) \leq r_{c}\right\}
$$

and

$$
\widetilde{I}_{c}=\varphi_{\triangleright_{c}}^{-1}(u) \cap I_{c}=\left\{i \in I_{c} \backslash \widehat{I}_{c}: \operatorname{rank}\left(i ; I \backslash \widehat{I}_{c}, \pi\right) \leq r_{u}\right\}
$$

as in a hard-reserves problem patients in $I_{c}$ are not eligible for any other category than $c$ and $u$ and patients in $I \backslash I_{c}$ are not eligible for $c$.

Let $i \in \varphi_{\triangleright_{c}}\left(I_{c}\right)$. Then there are two cases two consider. First consider the case that $i \in \widehat{I}_{c}$. If $\mu(i)=\emptyset$, then there exists $i^{\prime} \in \mu^{-1}(c) \backslash \varphi_{\triangleright_{c}}^{-1}(c)$ such that $i^{\prime} \pi i$ as $\mu$ respects priorities and is non-wasteful. By individual rationality of $\mu, i^{\prime} \in I_{c}$. These contradict the definition of $\widehat{I}_{c}$. Therefore, $\mu(i) \neq \emptyset$, which is equivalent to $i \in \mu\left(I_{c}\right)$.

Second consider the case that $i \in \widetilde{I}_{c}$. Suppose, for contradiction, that $\mu(i)=\emptyset$. Since $\mu$ is non-wasteful, $\left|\mu^{-1}(c)\right|=r_{c}$, and since $\mu$ respects priorities, for every $i^{\prime} \in \mu^{-1}(c), i^{\prime} \pi_{c} i$. Likewise, $\left|\mu^{-1}(u)\right|=r_{u}$, and since $\mu$ respects priorities, for every $i^{\prime} \in \mu^{-1}(u), i^{\prime} \pi_{u} i$. As 
a result, $\operatorname{rank}(i ; I, \pi)>r_{c}+r_{u}$, which is a contradiction to the construction of $\widehat{I}_{c}$ and $\widetilde{I}_{c}$. Therefore, $\mu(i) \neq \emptyset$, which is equivalent to $i \in \mu\left(I_{c}\right)$.

We conclude that $\varphi_{\triangleright_{c}}\left(I_{c}\right) \subseteq \mu\left(I_{c}\right)$.

Claim 2: $\mu\left(I_{c}\right) \subseteq \varphi_{\triangleright^{c}}\left(I_{c}\right)$.

Proof. We show that $\left|\mu\left(I_{c}\right)\right| \leq\left|\varphi_{\triangleright^{c}}\left(I_{c}\right)\right|$. The claim then follows because both $\mu$ and $\varphi_{\triangleright^{c}}$ respect priorities. Since both $\mu$ and $\varphi_{\triangleright^{c}}$ are non-wasteful, the inequality holds if, and only if, the number of category- $c$ beneficiaries assigned to unreserved units in $\mu$ is weakly less than the number of category- $c$ beneficiaries assigned to unreserved units in $\varphi_{\triangleright c}$ because we are considering a hardreserves rationing problem.

For every category $c^{\prime} \in \mathcal{C} \backslash\{u\}$, let

$$
\widehat{I}_{c^{\prime}}=\left\{i \in I_{c^{\prime}}: \operatorname{rank}\left(i ; I_{c^{\prime}}, \pi\right) \leq r_{c^{\prime}}\right\} .
$$

This is also the set of patients matched with any preferential treatment category $c^{\prime} \neq c$ in $\varphi_{\triangleright} c$ as all preferential treatments other than $c$ are processed before $u$ and $c$ in finding $\varphi_{\triangleright^{c}}$ and this is a hard-reserves problem.

Then the set of category-c beneficiaries matched to unreserved units in $\varphi_{\triangleright^{c}}$ is

$$
\widetilde{I}_{c}=\left\{i \in I_{c}: \operatorname{rank}\left(i ; I \backslash \cup_{c^{\prime} \in \mathcal{C} \backslash\{c, u\}} \widehat{I}_{c^{\prime}}, \pi\right) \leq r_{u}\right\}
$$

as the unreserved category $u$ is processed after all preferential treatment categories other than $c$ and before $c$ in finding $\varphi_{\triangleright^{c}}$.

Because $\mu$ is DA-induced by Theorem 1, the set of category-c beneficiaries matched to unreserved units in $\mu$ is

$$
\left\{i \in I_{c}: \operatorname{rank}\left(i ; I \backslash \cup_{c^{\prime} \in \mathcal{C} \backslash\{c, u\}} \mu^{-1}\left(c^{\prime}\right), \pi\right) \leq r_{u}\right\}
$$

The cardinality of this set is smaller than $\left|\widetilde{I}_{c}\right|$ because, by construction, $\left|\widehat{I}_{c^{\prime}}\right| \geq\left|\mu^{-1}\left(c^{\prime}\right)\right|$ and every patient in $\widehat{I}_{c^{\prime}} \backslash \mu^{-1}\left(c^{\prime}\right)$ has a higher priority according to $\pi$ than every patient in $\mu^{-1}\left(c^{\prime}\right) \backslash \widehat{I}_{c^{\prime}}$.

Proof of Theorem 5. Let $|\mathcal{C}| \leq 4$. Observe that the sequential reserve matchings $\varphi_{\triangleright}$ and $\varphi_{\triangleright^{\prime}}$ match the same patients to the categories with higher precedence than preferential treatment category $c$ and category $c^{\prime}$ under both $\triangleright$ and $\triangleright^{\prime}$. Let $J \subseteq I$ refer to the set of patients who are available when $c^{\prime}$ is about to be processed under $\triangleright^{\prime}$ (or equivalently when $c$ is about to be processed under $\triangleright$ ) in the sequential reserve matching procedure.

Observe that if $r_{c} \geq\left|J_{c}\right|$, then $\varphi_{\triangleright}\left(I_{c}\right)=\varphi_{\triangleright^{\prime}}\left(I_{c}\right)=I_{c}$ and we are done with the proof. Therefore, assume $r_{c}<\left|J_{c}\right|$.

Two cases are possible regarding category $c^{\prime}$, either $r_{c^{\prime}} \leq\left|J_{c^{\prime}}\right|$ or $r_{c^{\prime}}>\left|J_{c^{\prime}}\right|$.

First, suppose that $r_{c^{\prime}} \leq\left|J_{c^{\prime}}\right|$. Since both categories $c$ and $c^{\prime}$ have at least as many available beneficiary patients as their numbers of reserved units and give higher priority to their beneficiaries than every other patient, their capacity will be filled with the same patients among their beneficiaries according to priority orders $\pi_{c}$ and $\pi_{c^{\prime}}$, respectively, under both $\triangleright$ and $\triangleright^{\prime}$. Thus, 
the rest of the categories will be filled with the same patients, as well, and hence, category-c beneficiaries who are assigned a unit are identical under both matchings: $\varphi_{\triangleright}\left(I_{c}\right)=\varphi_{\triangleright^{\prime}}\left(I_{c}\right)$.

Next, suppose that $r_{c^{\prime}}>\left|J_{c^{\prime}}\right|$. Then $\left|J_{c^{\prime}}\right|$ units of category $c^{\prime}$ are assigned to category-c beneficiaries in $\varphi_{\triangleright}$ and $\varphi_{\triangleright^{\prime}}$ first. The rest of its capacity, which is $r_{c^{\prime}}-\left|J_{c^{\prime}}\right|$, is filled with respect to priority order $\pi$ conditional on the eligibility of patients for category $c^{\prime}$.

Recall that $\pi_{c}$ prioritizes category- $c$ beneficiaries over other patients. Thus, just before we process $c^{\prime}$ in the sequential reserve matching procedure under $\triangleright^{\prime}$, the highest priority $r_{c}$ patients in $J_{c}$ according to $\pi$ are no longer available and are matched with $c$. On the other hand when $c^{\prime}$ is about to be processed under $\triangleright$, the whole set $J_{c}$ is available. Under each order of precedence, since the remaining $r_{c^{\prime}}-\left|J_{c^{\prime}}\right|$ category-c $c^{\prime}$ units are allocated according to the baseline priority order $\pi$ to all eligible and available patients in $J \backslash J_{c^{\prime}}{ }^{23}$

$$
\begin{aligned}
& \varphi_{\triangleright^{\prime}}{ }^{-1}\left(\left\{c^{\prime}, c\right\}\right) \cap J_{c} \subseteq \varphi_{\triangleright}{ }^{-1}\left(\left\{c^{\prime}, c\right\}\right) \cap J_{c}, \\
& \varphi_{\triangleright}{ }^{-1}\left(c^{\prime}\right) \backslash \varphi_{\triangleright^{\prime}}{ }^{-1}\left(c^{\prime}\right) \subseteq J_{c} .
\end{aligned}
$$

To the contrary of what we are trying to prove, suppose there is a patient $i_{1} \in \varphi_{\triangleright^{\prime}}\left(J_{c}\right) \backslash \varphi_{\triangleright}\left(J_{c}\right)$. By Relationship (3), for some category $c_{1} \notin\left\{c, c^{\prime}\right\}$, we have $\varphi_{\triangleright^{\prime}}\left(i_{1}\right)=c_{1}$ while $\varphi_{\triangleright}\left(i_{1}\right)=\emptyset$. Hence, there are at least 3 categories in $\mathcal{C}$.

Since $\varphi_{\triangleright^{\prime}}\left(i_{1}\right)=c_{1}$ and $\varphi_{\triangleright^{\prime}}$ is individually rational, $i_{1}$ is eligible for $c_{1}$. Since $i_{1}$ is still unmatched after $c_{1}$ is processed under $\triangleright$,

$$
\left|\varphi_{\triangleright}^{-1}\left(c_{1}\right)\right|=r_{c_{1}},
$$

and there exists some patient $i_{2}$ such that

$$
i_{2} \pi_{c_{2}} i_{1} \quad \text { and } \quad \varphi_{\triangleright}\left(i_{2}\right)=c_{1} .
$$

Moreover, $i_{2}$ has to be matched with a category $c_{2}$ processed before $c_{1}$ under the sequential reserve matching procedure induced by $\triangleright^{\prime}$, so that $i_{1}$ is able to be matched with $c_{1}$ :

$$
c_{2} \triangleright^{\prime} c_{1}
$$

We also have $c_{2} \triangleright c_{1}$ as $c_{1} \notin\left\{c, c^{\prime}\right\}$.

Next, consider patient $i_{2}$ who is eligible for $c_{2}$ as $\varphi_{\triangleright^{\prime}}$ is individually rational. Since $i_{2}$ is still unmatched after $c_{2}$ is processed under $\triangleright$,

$$
\left|\varphi_{\triangleright}^{-1}\left(c_{2}\right)\right|=r_{c_{2}},
$$

and there exists some patient $i_{3}$ such that

$$
i_{3} \pi_{c_{2}} i_{2} \quad \text { and } \quad \varphi_{\triangleright}\left(i_{3}\right)=c_{2} .
$$

Moreover, $i_{3}$ has to be matched with a category $c_{3}$ that is processed before $c_{2}$ under sequential reserve matching procedure induced by $\triangleright^{\prime}$ so that $i_{2}$ is matched with $c_{2}$ :

$$
c_{3} \triangleright^{\prime} c_{2} \text {. }
$$

This leads to two cases:

\footnotetext{
${ }^{23}$ For any matching $\mu \in \mathcal{M}$ and set of categories $\mathcal{C}^{*} \subseteq \mathcal{C}$, recall that $\mu^{-1}\left(\mathcal{C}^{*}\right) \subseteq I$ is the set of patients matched to the categories in $\mathcal{C}^{*}$.
} 
Case 1. $c_{3} \triangleright c_{2}$ : Then $c_{2} \notin\left\{c, c^{\prime}\right\}$. Since $i_{3}$ is still unmatched after $c_{3}$ is processed under sequential reserve matching procedure induced by $\triangleright$,

$$
\left|\varphi_{\triangleright}^{-1}\left(c_{3}\right)\right|=r_{c_{3}},
$$

and there exists some patient $i_{4}$ such that

$$
i_{4} \pi_{c_{3}} i_{3} \quad \text { and } \varphi_{\triangleright}\left(i_{4}\right)=c_{3} .
$$

Moreover, $i_{4}$ has to be matched with a category $c_{4}$ that is processed before $c_{3}$ under the sequential reserve matching procedure induced by $\triangleright^{\prime}$, so that $i_{3}$ is able to be matched with $c_{3}$ :

$$
c_{4} \triangleright^{\prime} c_{3}
$$

If $c_{4} \triangleright c_{3}$, then $c_{3} \notin\left\{c, c^{\prime}\right\}$. Then there are at least five distinct categories ordered according to $\triangleright$ as $c^{\prime} \triangleright c \triangleright c_{3} \triangleright c_{2} \triangleright c_{1}$, which is a contradiction to $|\mathcal{C}| \leq 4$.

Therefore, $c_{3} \triangleright c_{4},|\mathcal{C}|=4$, and hence,

$$
c_{3}=c^{\prime} \quad \text { and } \quad c_{4}=c .
$$

Moreover, after $c$ is processed under $\triangleright, i_{1} \in J_{c}$ remains unmatched. Hence,

$$
\left|\varphi_{\triangleright}^{-1}(c)\right|=r_{c} .
$$

So far we have additionally $\left|\varphi_{\triangleright}^{-1}\left(c_{2}\right)\right|=r_{c_{2}},\left|\varphi_{\triangleright}^{-1}\left(c_{1}\right)\right|=r_{c_{1}}$, and $\left|\varphi_{\triangleright}^{-1}\left(c^{\prime}\right)\right|=r_{c^{\prime}}$. Yet $i_{1}$ is not matched under $\varphi_{\triangleright}$ and is matched under $\varphi_{\triangleright^{\prime}}$. This implies there exists some patient $i^{*} \in \varphi_{\triangleright}(J) \backslash \varphi_{\triangleright^{\prime}}(J)$. Since $\left\{i_{1}, i_{2}, i_{3}, i_{4}\right\} \subseteq \varphi_{\triangleright^{\prime}}(J), i^{*}$ is distinct from these four patients.

Let $\varphi_{\triangleright}\left(i^{*}\right)=c^{*}$ for some $c^{*} \in \mathcal{C}$.

As $i_{1}$ is still unmatched after $c^{*}$ is processed under the sequential reserve matching procedure induced by $\triangleright, i^{*} \pi_{c^{*}} i_{1}$. On the other hand, $i^{*}$ is still unmatched after $c_{1}$ is processed under the sequential reserve matching procedure induced by $\triangleright^{\prime}, i_{1} \pi_{c_{1}} i^{*}$. The last two statements together with $i_{1} \in J_{c}$ and $c \neq c_{1}$ imply (i) $i^{*} \notin J_{c}$, (ii) $c_{1}=u$ or $i^{*} \notin J_{c_{1}}$, (iii) $i_{1} \pi i^{*}$, and (iv) $c^{*} \neq c$.

Since $i^{*} \pi_{c^{*}} i_{1}, i^{*} \in J_{c^{*}}$ and $c^{*} \neq u$. Therefore, $c^{*} \in\left\{c^{\prime}, c_{2}\right\}$ as there are only 4 categories $c, c^{\prime}, c_{2}, c_{1}$. We consider these two cases separately, each of which will lead to a contradiction to the existence of category $c^{*}$ and will show that Case 1 cannot hold.

- First assume $c^{*}=c^{\prime}$ : but then $i^{*} \in \varphi_{\triangleright}^{-1}\left(c^{\prime}\right) \backslash \varphi_{\triangleright^{\prime}}^{-1}\left(c^{\prime}\right)$ despite the fact that $i^{*} \notin J_{c}$, contradicting Relationship (4). Thus, Case 1 cannot hold.

- Next assume $c^{*}=c_{2}$ : Recall that $\varphi_{\triangleright}\left(i_{2}\right)=c_{1}$ while $\varphi_{\triangleright^{\prime}}\left(i_{2}\right)=c_{2}$.

After $c_{2}$ is processed under the sequential reserve matching procedure induced by $\triangleright^{\prime}$, $i^{*}$ remains unmatched while $i_{2}$ is matched with $c_{2}$. Then we have

$$
i_{2} \pi_{c_{2}} i^{*}
$$


On the other hand, after $c_{2}$ is processed under the sequential reserve matching procedure induced by $\triangleright, i_{2}$ remains unmatched (because $i_{2}$ is matched with $c_{1}$, which is processed later since $c_{2} \triangleright c_{1}$ ), while $i^{*}$ is matched with $c_{2}$. Then it should be that

$$
i^{*} \pi_{c_{2}} i_{2}
$$

The last two displayed equations lead to a contradiction. Thus, Case 1 cannot hold.

Case 2. $c_{2} \triangleright c_{3}$ : Then $c_{3}=c$ and $c_{2}=c^{\prime}$. So far we have $\varphi_{\triangleright}\left(i_{3}\right)=c^{\prime}$ and $\varphi_{\triangleright^{\prime}}\left(i_{3}\right)=c$, hence we should have $i_{3} \in J_{c}$ as well by Relationship (4). Since $\varphi_{\triangleright^{\prime}}\left(i_{2}\right)=c^{\prime}$ and $\varphi_{\triangleright}\left(i_{2}\right)=c_{1} \neq c$, then by Relationship (3), $i_{2} \notin J_{c}$. Thus $i_{3} \pi_{c^{\prime}} i_{2}$ implies

$$
i_{3} \pi i_{2}
$$

Moreover, after $c$ is processed under the sequential reserve matching procedure induced by $\triangleright^{\prime}, i_{1} \in J_{c}$ remains unmatched while $i_{3} \in J_{c}$ is matched with it. Hence, $\left|\varphi_{\triangleright}^{-1}(c)\right|=r_{c}$.

After $c$ is processed under the sequential reserve matching procedure induced by $\triangleright, i_{1} \in J_{c}$ is not matched with it. Therefore, there exists some $j_{1} \in J_{c} \backslash\left\{i_{1}, i_{3}\right\}$ such that

$$
\varphi_{\triangleright}\left(j_{1}\right)=c, \quad \varphi_{\triangleright^{\prime}}\left(j_{1}\right) \notin\left\{c, c^{\prime}\right\}, \quad \text { and } \quad i_{3} \pi j_{1} \pi i_{1} .
$$

Moreover, since $j_{1}$ is still unmatched after $c^{\prime}$ is processed under the sequential reserve matching procedure under $\triangleright^{\prime}$, while $i_{2}$ is matched with $c^{\prime}, i_{2} \pi_{c^{\prime}} j_{1}$. Moreover, for $i_{3} \in J_{c}$ we have $i_{3} \pi_{c^{\prime}} i_{2}$, therefore $c^{\prime}$ is either unreserved or $i_{2} \notin J_{c^{\prime}}$ implying

$$
i_{2} \pi j_{1}
$$

Moreover, $j_{1}$ can be picked such that she is matched in a step before $i_{1}$ is matched with $c_{1}$ under the sequential reserve matching procedure induced by $\triangleright^{\prime} 24$

Since $c \triangleright^{\prime} c^{\prime} \triangleright^{\prime} c_{1}$ and $\varphi_{\triangleright^{\prime}}\left(j_{1}\right) \notin\left\{c_{1}, c, c^{\prime}\right\}$ then there is some category $c^{*}$ such that

$$
c \triangleright^{\prime} c^{\prime} \triangleright^{\prime} c^{*} \triangleright^{\prime} c_{1}
$$

and $\varphi_{\triangleright^{\prime}}\left(j_{1}\right)=c^{*}$. Hence, $|\mathcal{C}|=4$. Since $i_{2} \pi j_{1}$ and $i_{2}$ is still unmatched after $c^{*}$ is processed under the sequential reserve matching procedure induced by $\triangleright,\left|\varphi_{\triangleright}^{-1}\left(c^{*}\right)\right|=r_{c^{*}}$.

So far we have

$$
i_{3} \pi i_{2} \pi j_{1} \pi i_{1}
$$

\footnotetext{
${ }^{24}$ As otherwise: $\varphi_{\triangleright^{\prime}}\left(j_{1}\right)=c_{1}$ as $i_{1}$ cannot be matched before $j_{1}$. Then, the way we picked $i_{2}$ initially would be the replacement of $j_{1}$ in $\varphi_{\triangleright}$ as the patient who is matched with $c_{1}$ and we would have a second chain of agents and categories constructed for $i_{1}$ again and either Case 1 would eliminate this possibility or we would end up with this Case 2 again and at this point. Since the capacity of $c_{1}$ is finite, such iterations should end with such a $j_{1}$ as we picked in the text.
} 
and matchings $\varphi_{\triangleright}$ and $\varphi_{\triangleright^{\prime}}$ are, respectively, as follows:

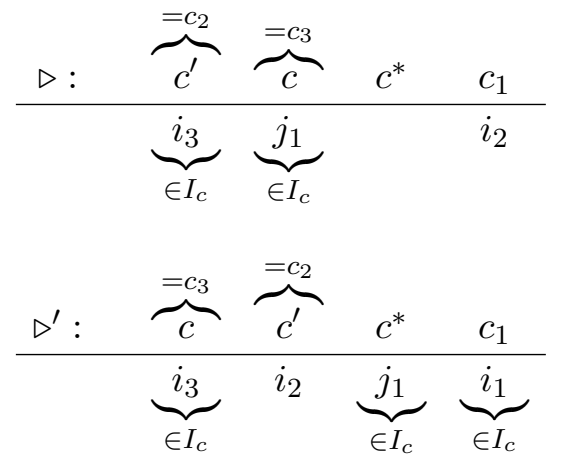

Observe from the above table that $i_{2}$ is not matched with $c^{*}$ after $c^{*}$ is processed the sequential reserve matching procedure induced by $\triangleright$, and yet a lower priority patient, $j_{1}$ is matched with $c^{*}$ under $\triangleright^{\prime}$. Then there should be some agent $j_{2}$ such that

$$
j_{2} \pi_{c^{*}} i_{2} \pi_{c^{*}} j_{1} \pi_{c^{*}} i_{1},
$$

and

$$
\varphi_{\triangleright}\left(j_{2}\right)=c^{*} \quad \text { and } \quad \varphi_{\triangleright^{\prime}}\left(j_{2}\right) \in\left\{c, c^{\prime}\right\} .
$$

If $\varphi_{\triangleright^{\prime}}\left(j_{2}\right)=c$, then $j_{2}$ should be matched with $c$ under $\triangleright$ instead of $j_{1}$, a contradiction. Thus, $\varphi_{\triangleright^{\prime}}\left(j_{2}\right)=c^{\prime}$. By Relationship (3), $j_{2} \notin J_{c}$.

Now notice that we are exactly at the same place when we first picked $j_{1}$. Then we will continue to construct a sequence of distinct patients $j_{1}, j_{2}, \ldots$

$$
\begin{aligned}
\ell \text { is odd } & \Longrightarrow j_{\ell} \in J_{c}, \quad \varphi_{\triangleright}\left(j_{\ell}\right)=c, \quad \varphi_{\triangleright^{\prime}}\left(j_{\ell}\right)=c^{*} ; \\
\ell \text { is even } & \Longrightarrow j_{\ell} \notin J_{c}, \quad \varphi_{\triangleright}\left(j_{\ell}\right)=c^{*}, \quad \varphi_{\triangleright^{\prime}}\left(j_{\ell}\right)=c^{\prime} .
\end{aligned}
$$

and

$$
\ell \text { is even } \Longrightarrow j_{\ell} \pi j_{\ell-1} \text {. }
$$

Since this sequence is infinite, this contradicts to the finiteness of the problem. Thus, Case 2 cannot hold.

Therefore, such a patient $i_{1}$ cannot exist, which equivalently means $\varphi_{\triangleright^{\prime}}\left(J_{c}\right) \subseteq \varphi_{\triangleright}\left(J_{c}\right)$. Since $\varphi_{\triangleright^{\prime}}\left(I_{c} \backslash J_{c}\right)=\varphi_{\triangleright}\left(I_{c} \backslash J_{c}\right)=I_{c} \backslash J_{c}$,

$$
\varphi_{\triangleright^{\prime}}\left(I_{c}\right) \subseteq \varphi_{\triangleright}\left(I_{c}\right),
$$

completing the proof of the theorem. 


\section{References}

Abdulkadiroğlu, Atila. 2005. "College Admissions with Affirmative Action." International Journal of Game Theory, 33(4): 535-549.

Abdulkadiroğlu, Atila, and Tayfun Sönmez. 2003. "School Choice: A Mechanism Design Approach." American Economic Review, 93: 729-747.

Agarwal, Nikhil, Itai Ashlagi, Michael Rees, Paulo Somaini, and Daniel Waldinger. 2019. "An Empirical Framework for Sequential Assignment: The Allocation of Deceased Donor Kidneys." Working paper, MIT.

Al-Jazeera. 2020. "COVID-19: US conducts trials of plasma transfusions." April 6.

Andersson, Tommy. 2017. "Refugee Matching as a Market Design Application." Working paper.

Aygün, Orhan, and Bertan Turhan. 2016. "Dynamic Reserves in Matching Markets." Working paper.

Aygün, Orhan, and Bertan Turhan. 2017. "Large-Scale Affirmative Action in School Choice: Admissions to IITs in India." American Economic Review, 107(5): 210-13.

Aygün, Orhan, and Inacio Bo. 2016. "College Admission with Multidimensional Reserves: The Brazilian Affirmative Action Case." Working paper, WZB Berlin Social Science Center, 869 .

Balinski, Michel, and Tayfun Sönmez. 1999. "A Tale of Two Mechanisms: Student Placement." Journal of Economic Theory, 84: 73-94.

Ball, Robert, and Phil Schneider. 2009. "South Carolina Prepares for Pandemic Influenza: An Ethical Perspective." South Carolina Pandemic Influenza Ethics Task Force.

Baswana, Surender, Partha Pratim Chakrabarti, Sharat Chandran, Yashodhan Kanoria, and Utkarsh Patange. 2018. "Centralized admissions for engineering colleges in India." forthcoming, INFORMS Journal on Applied Analytics.

Bateman, Scot, Monica Bharel, Paul Biddinger, Peter A. DePergola, Lachlan Forrow, Eric Goralnick, Joshua Kornbluth, Abraar Karan, Christine Mitchell, Emily Rubin, Dean Shapley, Sally Vitali, Michael Wagner, Matthias Walz, and Ellen Weinstein. 2020. "Crisis Standards of Care Planning Guidance for the COVID-19 Pandemic." April 7.

Biddison, Elizabeth, Howard Gwon, Monica Schoch-Spana, Robert Cavalier, Douglas B. White, Timothy Dawson, Peter B. Terry, Alex John London, Alan Regenberg, Ruth Faden, and Eric S. Toner. 2013. "The Community Speaks: Understanding Ethical Values in Allocation of Scarce Lifesaving Resources during Disasters." Annals of the American Thoracic Society, 11: 777-783. 
Biro, P., T. Fleiner, R.W. Irving, and D.F. Manlove. 2010. "The College Admissions Problem with Lower and Common Quotas." Theoretical Computer Science, 411(34-26): 31363153.

Blow, Charles. 2020. "The Racial Time Bomb in the Covid-19 Crisis." New York Times, April 1.

Bo, Inacio. 2016. "Fair Implementation of Diversity in School Choice." Games and Economic Behavior, 97: 54-63.

Budish, Eric. 2011. "The Combinatorial Assignment Problem: Approximate Competitive Equilibrium from Equal Incomes." Journal of Political Economy, 119(6): 1061-1103.

Budish, Eric, Yeon-Koo Che, Fuhito Kojima, and Paul R. Milgrom. 2013. "Designing Random Allocation Mechanisms: Theory and Applications." American Economic Review, 103(2): 585-623.

Carter, Stephen. 2020. "Ventilator Rationing Guidelines Are Discriminatory." Bloomberg, April 10.

Christian, M.D., L Hawryluck, R. S. Wax, T Cook, N. M. Lazar, M. S. Herridge, M. P. Muller, D. R. Gowans, W. Fortier, and F. M. Burkle. 2006. "Development of a triage protocol for critical care during an influenza pandemic." Canadian Medical Association Journal, 175: 1377-1381.

Correa, Jose, Rafael Epstein, Juan Escobar, Ignacio Rios, Bastian Bahamondes, Carlos Bonet, Natalie Epstein, Nicolas Aramayo, Martin Castillo, Andres Cristi, and Boris Epstein. 2019. "School Choice in Chile." EC '19, 325-343. New York, NY, USA:ACM.

Daugherty-Biddison, Lee, Howard Gwon, Alan Regenberg, Monica Schoch-Spana, and Eric Toner. 2017. "Maryland Framework for the Allocation of Scarce Life-Sustaining Medical Resources in a Catastrophic Public Health Emergency." $\quad$ https://www.law.umaryland.edu/media/SOL/pdfs/Programs/HealthLaw/MHECN/ASR\%20Framework_Final.pdf.

Delacrétaz, David, Scott Duke Kominers, and Alexander Teytelboym. 2016. "Refugee Resettlement." Working paper.

de Pu Kamp, Majlie, Curt Devine, and Drew Griffin. 2020. "As coronavirus cases grow, hospitals adopt a system to rank patients for treatment." CNN, April 3, https:// www.cnn.com/2020/04/03/health/coronavirus-hospital-ethics-ventilators-invs/ index.html.

Dittmar, Pia. 2018. "Gender Quotas - Balancing Act Between Efficient Policies and Gap Fillers." EU-Logos Athena. 
Dogan, Battal. 2016. "Responsive Affirmative Action in School Choice." Journal of Economic Theory, 165: 69-105.

DoH, Utah. 2018. "Utah Crisis Standards of Care Guidelines." Utah Department of Health.

DREDF. 2020. "Preventing Discrimination in the Treatment of Covid-19 Patients: The Illegality of Medical Rationing on the Basis of Disability." March 25, Available at https://dredf.org/wp-content/uploads/2020/03/ DREDF-Policy-Statement-on-COVID-19-and-Medical-Rationing-3-25-2020.pdf.

Duan, Kai, Bende Liu, Cesheng Li, Huajun Zhang, Ting Yu, Jieming Qu, Min Zhou, Li Chen, Shengli Meng, Yong Hu, Cheng Peng, Mingchao Yuan, Jinyan Huang, Zejun Wang, Jianhong Yu, Xiaoxiao Gao, Dan Wang, Xiaoqi Yu, Li Li, Jiayou Zhang, Xiao Wu, Bei Li, Yanping Xu, Wei Chen, Yan Peng, Yeqin Hu, Lianzhen Lin, Xuefei Liu, Shihe Huang, Zhijun Zhou, Lianghao Zhang, Yue Wang, Zhi Zhang, Kun Deng, Zhiwu Xia, Qin Gong, Wei Zhang, Xiaobei Zheng, Ying Liu, Huichuan Yang, Dongbo Zhou, Ding Yu, Jifeng Hou, Zhengli Shi, Saijuan Chen, Zhu Chen, Xinxin Zhang, and Xiaoming Yang. 2020. "Effectiveness of convalescent plasma therapy in severe COVID-19 patients." Proceedings of the National Academy of Sciences.

Dur, Umut, Parag Pathak, and Tayfun Sönmez. 2019. "Explicit vs. Statistical Preferential Treatment in Affirmative Action: Theory and Evidence from Chicago's Exam Schools." forthcoming, Journal of Economic Theory.

Dur, Umut, Scott Kominers, Parag Pathak, and Tayfun Sönmez. 2018. "Reserve Design: Unintended Consequences and The Demise of Boston's Walk Zones." Journal of Political Economy, 126(6).

Echenique, Federico, and M. Bumin Yenmez. 2015. "How to Control Controlled School Choice." American Economic Review, 105(8): 2679-2694.

Edelman, Benjamin, Michael Ostrovsky, and Michael Schwarz. 2007. "Internet Advertising and the Generalized Second-Price Auction: Selling Billions of Dollars Worth of Keywords." American Economic Review, 97(1): 242-259.

Ehlers, Lars, Isa E. Hafalir, M. Bumin Yenmez, and Muhammed A. Yildirim. 2014. "School Choice with Controlled Choice Constraints: Hard Bounds versus Soft Bounds." Journal of Economic Theory, 153: 648-683.

Emanuel, Ezekial J., Govind Persad, Ross Upshur, Beatriz Thome, Michael Parker, Aaron Glickman, Cathy Zhang, Conor Boyle, Maxwell Smith, and James Philips. 2020. "Fair Allocation of Scarce Medical Resources in the Time of Covid-19." New England Journal of Medicine, March 23.

Fink, Sheri. 2020. "U.S. Civil Rights Office Rejects Rationing Medical Care Based on Disability, Age." New York Times, March 29. 
Fragiadakis, Daniel, and Peter Troyan. 2017. "Improving Matching under Hard Distributional Constraints." Theoretical Economics, 12(2): 863-908.

Gale, David, and Lloyd S. Shapley. 1962. "College Admissions and the Stability of Marriage." American Mathematical Monthly, 69: 9-15.

Hafalir, Isa E., M. Bumin Yenmez, and Muhammed A. Yildirim. 2013. "Effective Affirmative Action in School Choice." Theoretical Economics, 8(2): 325-363.

Hsieh, Nien-hê. 2016. "Incommensurable Values." January 25, https://plato.stanford. edu/entries/value-incommensurable/\#Bib.

Israni, A. K., N. Salkowski, S. Gustafson, J. J. Snyder, J. J. Friedewald, R. N. Formica, X. Wang, E. Shteyn, W. Cherikh, D. Stewart, C. J. Samana, A. chung, A. Hart, and B. L. Kasiske. 2014. "New National Allocation Policy for Deceased Donor Kidneys in the United States and Possible Effects on Patient Outcomes." Journal of the American Society of Nephrology, 25(8): 1842-1848.

Iten, Anne, and Pascal Strupler. 2018. "Swiss Influenza Pandemic Plan." Federal Office of Public Health, Swiss Confederation.

Jones, A. E., S. Trzeciak, and J. A. Kline. 2009. "The Sequential Organ Failure Assessment score for predicting outcome in patients with severe sepsis and evidence of hypoperfusion at the time of emergency department presentation." Critical Care Medicine, 37: 1649-1654.

Jones, Will, and Alexander Teytelboym. 2017. "The Local Refugee Match: Aligning Refugees' Preferences with the Capacities and Priorities of Localities." Journal of Refugee Studies, 31(2): 152-178.

Kamada, Yuichiro, and Fuhito Kojima. 2015. "Efficient Matching under Distributional Constraints: Theory and Applications." American Economic Review, 105(1): 67-99.

Kamada, Yuichiro, and Fuhito Kojima. 2017. "Stability Concepts in Matching with Distributional Constraints." Journal of Economic Theory, 168: 107-142.

Kamada, Yuichiro, and Fuhito Kojima. 2018. "Stability and Strategy-proofness for Matching with Constraints: A Necessary and Sufficient Condition." Theoretical Economics, 13(2): 761-794.

Kojima, Fuhito. 2012. "School Choice: Impossibilities for Affirmative Action." Games and Economic Behavior, 75(2): 685-693.

Kominers, Scott Duke, and Tayfun Sönmez. 2016. "Matching with Slot-specific Priorities: Theory." Theoretical Economics, 11(2): 683-710.

Michigan. 2012. "Guidelines for Ethical Allocation of Scarce Medical Resources and Services During Public Health Emergencies in Michigan." Department of Community Health, Office of Public Health Preparedness. 
Milgrom, Paul R. 2000. "Putting Auction Theory to Work: The Simultaneous Ascending Auction." Journal of Political Economy, 108: 245-272.

Ne’eman, Ari. 2020. "I Will Not Apologize for My Needs." New York Times, March 23.

OPTN. 2014. "Organ Procurement and Transplantation Network Policies." Technical Report.

Pathak, Parag A., and Tayfun Sönmez. 2008. "Leveling the Playing Field: Sincere and Sophisticated Players in the Boston Mechanism." American Economic Review, 98(4): 16361652.

Pathak, Parag A., and Tayfun Sönmez. 2013a. "School Admissions Reform in Chicago and England: Comparing Mechanisms by their Vulnerability to Manipulation." American Economic Review, 103(1): 80-106.

Pathak, Parag, Alex Rees-Jones, and Tayfun Sönmez. 2020a. "Immigration Lottery Design: Engineered and Coincidental Consequences of H-1B Reforms." NBER Working Paper 26767.

Pathak, Parag, Alex Rees-Jones, and Tayfun Sönmez. 2020b. "Reversing Reserves." NBER Working Paper.

Pathak, Parag, and Tayfun Sönmez. 2013b. "Recommendation on Algorithm Processing, Public Testimony to EAC." Available at: https://economics.mit.edu/files/16966.

Persad, Govind. 2020. "Why Disability Law Permits Evidence-Based Triage in a Pandemic." forthcoming, Yale Law Journal Online.

Rankin, Jennifer. 2020. "EU revises plans for mandatory quotas of women on company boards." The Guardian, March 5.

Roth, Alvin E. 1984. "The Evolution of the Labor Market for Medical Interns and Residents: A Case Study in Game Theory." Journal of Political Economy, 92: 991-1016.

Roth, Alvin E., and Elliott Peranson. 1999. "The Redesign of the Matching Market for American Physicians: Some Engineering Aspects of Economic Design." American Economic Review, 89: 748-780.

Roth, Alvin E., Tayfun Sönmez, and Utku Ünver. 2004. "Kidney Exchange." Quarterly Journal of Economics, 119: 457-488.

Roth, Alvin E., Tayfun Sönmez, and Utku Ünver. 2005. "Pairwise Kidney Exchange." Journal of Economic Theory, 125: 151-188.

Schummer, James, and Azar Abizada. 2017. "Incentives in Landing Slot Problems." Journal of Economic Theory, 170: 29-55.

Schummer, James, and Rakesh V. Vohra. 2013. "Assignment of Arrival Slots." American Economic Journal: Microeconomics, 5(2): 164-185. 
Sönmez, Tayfun. 2013. "Bidding for Army Career Specialties: Improving the ROTC Branching Mechanism." Journal of Political Economy, 121(1): 186-219.

Sönmez, Tayfun, and M. Bumin Yenmez. 2019a. "Affirmative Action in India via Vertical and Horizontal Reservations." Working paper.

Sönmez, Tayfun, and M. Bumin Yenmez. 2019b. "Constitutional Implementation of Vertical and Horizontal Reservations in India: A Unified Mechanism for Civil Service Allocation and College Admissions." Working paper.

Sönmez, Tayfun, and M. Utku Ünver. 2010. "Course Bidding at Business Schools." International Economic Review, 51(1): 99-123.

Sönmez, Tayfun, and Tobias Switzer. 2013. "Matching with (Branch-of-Choice) Contracts at the United States Military Academy." Econometrica, 81(2): 451-488.

Sönmez, Tayfun, Utku Ünver, and M. Bumin Yenmez. 2020. "Incentivized Kidney Exchange." forthcoming, American Economic Review.

Tong, Rosemarie, and Leah Devlin. 2007. "Stockpiling Solutions: North Carolina's Ethical Guidelines for an Influenza Pandemic." North Carolina Institute of Medicine, Department of Health and Human Services Division of Public Health.

Truog, Robert D., Christine Mitchell, and George Q. Daley. 2020. "The Toughest Triage - Allocating Ventilators in a Pandemic." New England Journal of Medicine, March 23.

Varian, Hal R. 2007. "Position Auctions." International Journal of Industrial Organization, 25(6): 1163-1178.

Vawter, Dorothy E., J. Eline Garrett, Karen G. Gervais, Angela Witt Prehn, Debra A. DeBruin, Carol A. Tauer, Elizabeth Parilla, Joan Liaschenko, and Mary Faith Marshall. 2010. "For the Good of Us All: Ethically Rationing Health Resources in Minnesota in a Severe Influenza Pandemic." Minnesota Pandemic Ethics Project, Minnesota Department of Health.

Vincent, J. L., R. Moreno, J. Takala, S. Willatts, A. De Mendonça, H. Bruining, C. K. Reinhart, P. M. Suter, and L. G. Thijs. 1996. "The SOFA (Sepsis-related Organ Failure Assessment) score to describe organ dysfunction/failure. On behalf of the Working Group on Sepsis-Related Problems of the European Society of Intensive Care Medicine." Intensive Care Medicine, 22: 707-710.

Westkamp, Alexander. 2013. "An Analysis of the German University Admissions System." Economic Theory, 53(3): 561-589.

White, Douglas B., and Bernard Lo. 2020. "A Framework for Rationing Ventilators and Critical Care Beds During the Covid-19 Pandemic." Journal of the American Medical Association. doi:10.1001/jama.2020.5046. 
White, Douglas B., Mitchell H. Katz, John M. Luce, and Bernard Lo. 2009. "Who Should Receive Life Support During a Public Health Emergency? Using Ethical Principles to Improve Allocation Decisions." Annals of Internal Medicine, 150(2): 132-138.

White, Douglas B., Mithcell Katz, John M. Luce, and Bernard Lo. 2020. "Allocation of Scarce Critical Care Resources During a Public Health Emergency." University of Pittsburgh, Department of Critical Care Medicine, March 26.

Whyte, Liz Essley. 2020. "State Policies May Send People with Disabilities to the Back of the Line for Ventilators." April 8, https://publicintegrity.org/health/coronavirus-and-inequality/ state-policies-may-send-people-with-disabilities-to-the-back-of-the-line-for-ventilators/

Zucker, Howard A, Karl P. Adler, Donald P. Berens, Rock Brynner, Karen Butler, Yvette Calderon, Carolyn Corcoran, Nancy Neveloff Dubler, Paul J. Edelson, Joseph J. Fins, Rev. Francis H. Geer, Samuel Gorovitz, Cassandra Henderson, Hassan Khouli, Fr. Joseph W. Koterski, Hugh Maynard-Reid, John Murnane, Karen Porter, Robert Swidler, Sally True, Stuart C. Sherman, Susia A. Han, and Valier Gutmann Koch. 2015. "Ventilator Allocation Guidelines." New York State Task Force on Life and the Law, New York Department of Health. 